\title{
Introduction to dynamical horizons in numerical relativity
}

\author{
Erik Schnetter, ${ }^{1,2, *}$ Badri Krishnan, ${ }^{2, \dagger}$ and Florian Beyer ${ }^{2, \ddagger}$ \\ ${ }^{1}$ Center for Computation and Technology, 302 Johnston Hall, Louisiana State University, Baton Rouge, Louisiana 70803, USA ${ }^{\S}$ \\ ${ }^{2}$ Max-Planck-Institut für Gravitationsphysik, Albert-Einstein-Instituto, Am Mühlenberg 1, D-14476 Golm, Germany
}

(Received 11 April 2006; published 28 July 2006)

This paper presents a quasilocal method of studying the physics of dynamical black holes in numerical simulations. This is done within the dynamical horizon framework, which extends the earlier work on isolated horizons to time-dependent situations. In particular: (i) We locate various kinds of marginal surfaces and study their time evolution. An important ingredient is the calculation of the signature of the horizon, which can be either spacelike, timelike, or null. (ii) We generalize the calculation of the black hole mass and angular momentum, which were previously defined for axisymmetric isolated horizons to dynamical situations. (iii) We calculate the source multipole moments of the black hole which can be used to verify that the black hole settles down to a Kerr solution. (iv) We also study the fluxes of energy crossing the horizon, which describes how a black hole grows as it accretes matter and/or radiation. We describe our numerical implementation of these concepts and apply them to three specific test cases, namely, the axisymmetric head-on collision of two black holes, the axisymmetric collapse of a neutron star, and a nonaxisymmetric black hole collision with nonzero initial orbital angular momentum.

DOI: 10.1103/PhysRevD.74.024028

\section{INTRODUCTION}

In spite of fundamental advances in our understanding of black holes, relatively little is known about them in the fully nonperturbative, dynamical regime of general relativity. Most of our intuition regarding black holes comes from studying the stationary, axisymmetric Kerr-Newman solutions, and perturbations thereof. This, along with postNewtonian calculations which treat the black hole as a point particle, are usually adequate for understanding many astrophysical processes involving black holes. However, understanding the gravitational waveforms arising due to, say, the merger phase of the coalescence of two black holes or the gravitational collapse of a star, will require us to go beyond perturbation theory and to confront the nonlinearities and dynamics of the full Einstein equations. This regime may contain qualitatively new, nonperturbative features. In this paper, we discuss an important ingredient for understanding this regime, namely, the dynamics of the black hole horizon. Numerical simulations of black holes have greatly improved in the last few years. Simulations of the entire merger process, starting from the last few orbits of the inspiral right up to the ringdown have become possible in the past year [1-8]. It is then important to look for better ways to extract more physical information from simulations and to compare results from two different simulations performed using different coordinate systems, gauge conditions, etc. This can be a nontrivial task in itself, and understanding black hole horizons is a necessary ingredient.

\footnotetext{
*Electronic address: schnetter@cct.lsu.edu

†Electronic address: badri.krishnan@aei.mpg.de

*Electronic address: florian.beyer@aei.mpg.de

${ }^{\S} \mathrm{URL}$ : http://www.cct.lsu.edu/about/focus/numerical/

"URL: http://numrel.aei.mpg.de/

URL: http://www.aei.mpg.de/
}

PACS numbers: 04.25.Dm, 04.70.Bw, 95.30.Sf, 97.60.Lf

Because of their global nature, black hole event horizons can only be located once a simulation is complete and we have obtained the full spacetime. In numerical simulations, it is instead common to use marginally trapped surfaces to locate black holes on a Cauchy surface in real time. We use the formalism of dynamical horizons $[9,10]$ to study black holes. Using isolated/dynamical horizons, it is shown that marginally trapped surfaces, while not a substitute for event horizons, do have many useful properties and can be used fruitfully to study black hole physics. Dynamical horizons are a significant extension of the isolated horizon framework [11-15], which models isolated stationary black holes in an otherwise dynamical spacetime. Both these frameworks are, in turn, very closely related to and motivated by the earlier work on trapping horizons by Hayward [16-18]. See [19-21] for reviews. Information obtained from these quasilocal horizons complements the information obtained from the event horizon. Once a simulation is complete and ready for post-processing, event horizons are useful for studying global properties and the causal structure of the spacetime, and also phenomena such as the topology change of the horizon during a black hole coalescence. Reliable and computationally efficient codes are now available for locating event horizons (see e.g. [22]). Such information cannot be obtained at the quasilocal level, which is instead better for tracking the physical parameters and geometry of a black hole in real time.

The dynamics of apparent and event horizons have been numerically studied in the past in detail in axisymmetry (see e.g. [23-28]). We want to extend this work to nonaxisymmetric and nonvacuum spacetimes, and we want to emphasize non-gauge-dependent analysis methods. In particular, we consider the following applications: (i) We study the behavior of various marginally trapped surfaces under time evolution. This leads to greater insights about the trapped region of a spacetime. An important ingredient 
here is the signature of the world tube of marginally trapped surfaces. This world tube is known to be null for isolated horizons, and more generally, it can be either spacelike or timelike; we show that both types occur frequently in numerical simulations. (ii) We give meaningful definitions for the angular momentum, mass, and higher multipole moments for the dynamical black hole. The multipole moments capture gauge invariant geometrical information regarding the horizon geometry, and should be useful for understanding fundamental issues such as the final state of black hole collapse. For example, we would expect that after a black hole has formed and settled down, its multipole moments should be identical to the source multipoles of a Kerr black hole. We show that it is, in principle, possible to verify this conjecture and to calculate the rate at which a black hole approaches equilibrium. (iii) We also describe and implement methods for calculating the energy flux falling into the horizon. This gives us detailed information on how black holes grow as they swallow matter and radiation.

This paper is organized as follows. Section II sets up notation, and summarizes the basic definitions and properties of trapped surfaces and dynamical horizons. Section III describes the various physical quantities that we calculate using dynamical horizons, and also their numerical implementation. Section IV presents three concrete, well-known numerical examples where these concepts are applied and finally, Sec. V discusses some open issues and directions for further work. Unless mentioned otherwise, we use geometrical units with $G=c=1$, the spacetime signature is $(-,+,+,+)$, all manifolds and fields are assumed to be smooth, and the Penrose abstract index notation is used throughout. The derivative operator compatible with the spacetime metric $g_{a b}$ is $\nabla_{a}$ and, following Wald [29], the Riemann tensor is defined via $\left(\nabla_{a} \nabla_{b}-\nabla_{b} \nabla_{a}\right) \omega_{c}=R_{a b c}{ }^{d} \omega_{d}$.

\section{BASIC NOTIONS AND DEFINITIONS}

\section{A. Trapped surfaces and apparent horizons}

Let $S$ be a closed, orientable spacelike 2-surface in a 4dimensional spacetime $\left(\mathcal{M}, g_{a b}\right)$. The expansion of any such surface can be defined invariantly without any reference to a time slicing of the spacetime. Since $S$ is smooth, spacelike, and 2-dimensional, the set of vectors orthogonal to it at any point form a 2-dimensional Minkowskian vector space. Thus, we can define two linearly independent, future-directed, null vectors $\ell^{a}$ and $n^{a}$ orthogonal to $S$ such that

$$
g_{a b} \ell^{a} n^{b}=-1 .
$$

Note that this convention is different from that used in [10]. We shall assume that we know a priori what the outgoing and ingoing directions on $\mathcal{M}$ are. By convention, $\ell^{a}$ will denote an outgoing null normal and $n^{a}$ an ingoing one. The null normals are specified only up to a boost transformation

$$
\ell^{a} \rightarrow f \ell^{a}, \quad n^{a} \rightarrow f^{-1} n^{a}
$$

where $f$ is a, positive definite, smooth function on $S$. All physical quantities must be invariant under this gauge transformation.

The Riemannian 2-metric $\tilde{q}_{a b}$ on $S$ induced by the spacetime metric $g_{a b}$ is

$$
\tilde{q}_{a b}=g_{a b}+\ell_{a} n_{b}+n_{a} \ell_{b} .
$$

The tensor $\tilde{q}_{a}^{b}$ can be viewed as a projection operator on to $S$. The null expansions are

$$
\Theta_{(\ell)}=\tilde{q}^{a b} \nabla_{a} \ell_{b}, \quad \Theta_{(n)}=\tilde{q}^{a b} \nabla_{a} n_{b} .
$$

These expansions tell us how the area element of $S$ changes as it is deformed along $\ell^{a}$ and $n^{a}$ respectively.

The shear of $\ell^{a}, \sigma_{(\ell) a b}$, is the symmetric trace-free part of the projection of $\nabla_{a} \ell_{b}$ :

$$
\sigma_{(\ell) a b}=\tilde{q}_{a}^{c} \tilde{q}_{b}^{d} \nabla_{(c} \ell_{d)}-\frac{1}{2} \Theta_{(\ell)} \tilde{q}_{a b} .
$$

Similarly, the shear of $n^{a}$ is

$$
\sigma_{(n) a b}=\tilde{q}_{a}^{c} \tilde{q}_{b}^{d} \nabla_{(c} n_{d)}-\frac{1}{2} \Theta_{(n)} \tilde{q}_{a b} .
$$

Note that these definitions only involve derivatives tangential to $S$. Thus $\ell^{a}$ and $n^{a}$ can, if necessary, be extended arbitrarily away from $S$ while computing these quantities.

The closed 2-surface $S$ is said to be a trapped surface if both expansions $\Theta_{(\ell)}$ and $\Theta_{(n)}$ are strictly negative. This is very different from a sphere in normal flat space which has positive outgoing expansion and negative ingoing expansion. This definition was first introduced by Penrose [30], who recognized its importance in the formation of singularities. On a marginal surface, one of the two null expansions vanish. Of particular interest are the marginally outer trapped surfaces (MOTSs), for which the outgoing null rays along $\ell^{a}$ have zero expansion. In addition, we shall mostly deal with future marginally outer trapped surfaces (FMOTSs), i.e., MOTSs with $\Theta_{(n)}<0$.

There are three main reasons why closed trapped surfaces are important for studying black holes. First, the existence of a trapped surface implies the existence of a singularity in the future [30,31]. Second, they are guaranteed to always lie within the event horizon. Finally, in stationary spacetimes, the null generators of the event horizon have zero expansion. Thus for stationary spacetimes, the cross section of the event horizon is a MOTS.

While trapped and marginally outer trapped surfaces are defined in the full four dimensional spacetime, in numerical relativity, one usually considers trapped surfaces in conjunction with a foliation of (partial) Cauchy surfaces containing $S$; it is numerically much easier to look for closed surfaces on the Cauchy surface rather than in the full spacetime manifold. For concreteness, we shall work in the ADM formalism where the relevant portion of spacetime is foliated by spacelike surfaces, and $\Sigma$ shall denote one of the leaves of this foliation. However, it will be 
obvious that the formalism is applicable no matter how Einstein's equations are implemented.

The trapped region $\mathcal{T}_{\Sigma}$ on $\Sigma$ is defined to be the set of points in $\Sigma$ through which there passes a trapped surface contained entirely in $\Sigma$. Note that there could be points in $\Sigma$ not contained in $\mathcal{T}_{\Sigma}$, but through which there passes a trapped surface not contained in $\Sigma$. Thus, $\mathcal{T}_{\Sigma}$ is a subset of the intersection of $\Sigma$ with the 4-dimensional trapped region in the full spacetime. A connected component of the boundary of $\mathcal{T}_{\Sigma}$ is called an apparent horizon (AH). Under suitable regularity conditions, the $\mathrm{AH}$ can be shown to be a MOTS $[32,33]$. Thus, an apparent horizon is the outermost MOTS on $\Sigma$. Because of this "outermost" property, an $\mathrm{AH}$ is not a quasilocal object on $\Sigma$. The behavior of AHs under time evolution can be quite irregular. For example, they can "jump" discontinuously. On the other hand, as we shall soon see, MOTSs are more regular.

\section{B. Dynamical horizons}

\section{Definition and examples}

We can use marginal surfaces to extract physically interesting information about the black hole. The key idea is to look not at a single MOTS by itself, but rather a world tube $H$ of MOTSs constructed by stacking up the MOTSs obtained by time evolution. Such a world tube is called a marginally trapped tube (MTT). A MTT is thus a smooth 3-surface foliated by MOTSs.

The existence of MTTs: Numerically, it has been observed that marginal surfaces (though not apparent horizons - see below) usually behave smoothly under time evolution and produce a smooth MTT. This observation is placed on a more rigorous footing by the recent result of Andersson et al. [34], which proves the local existence of MTTs for a large class of MOTSs. Their results require the MOTS to be strictly-stably-outermost. An MOTS $S$ on $\Sigma$ is said to be strictly-stably-outermost if there exists an infinitesimal first order outward deformation which makes $S$ strictly untrapped. Working with a radial coordinate $r$ on $\Sigma$ such that $S$ is a level set of $r$, and $r$ increases in the outward direction, a sufficient (but not necessary) condition for $S$ to be strictly-stably-outermost is $\partial_{r} \Theta_{(\ell)}(r)>0$ everywhere $^{1}$ on $S$. Here it is understood that we obtain $\Theta_{(\ell)}$ as a function of $r$ by calculating $\Theta_{(\ell)}$ for the constant $r$ surfaces in the vicinity of $S$. In principle, for an unfortunate choice of $r$, it might happen that $\partial_{r} \Theta_{(\ell)}<0$ even though there is a different choice for which this condition is satisfied. In any case, this is sufficient for verifying that $S$ is strictly-stably-outermost. ${ }^{2}$ This condition, unlike the

\footnotetext{
${ }^{1}$ More precisely, $\partial_{r} \Theta_{(\ell)}(r) \geq 0$ with $\partial_{r} \Theta_{(\ell)}(r)>0$ somewhere on $S$.

${ }^{2}$ It is harder to show that a MOTS is not strictly-stablyoutermost. This can be done by calculating the signature of the horizon (see below) or by calculating the principle eigenvalue of the stability operator defined in [34].
}

outermost condition for an $\mathrm{AH}$, is a quasilocal condition. We have found in our simulations that most physically interesting MOTSs, such as ones which asymptote to the event horizon, and also AHs, satisfy this condition quite generally. However, as we shall see, there exist also MOTSs which are not strictly-stably-outermost. In practice, instead of checking $\partial_{r} \Theta_{(\ell)}>0$ directly, we look for a surface with a small positive (or negative) nonvanishing expansion, and check that it lies completely outside (or inside) the MOTS.

It is shown in [34] that if a MOTS $S$ is strictly-stablyoutermost, then at least locally in time, $S$ is a cross section of a smooth MTT. More explicitly, this result shows that given a foliation of the spacetime by Cauchy surfaces $\Sigma_{t}$, if there is a MOTS $S_{0}$ on $\Sigma_{0}$ which is strictly-stablyoutermost, then MOTSs $S_{t}$ exist on $\Sigma_{t}$ for $-\epsilon<t<\epsilon$ (for sufficiently small $\epsilon$ ) such that the union $\bigcup S_{t}$ is a smooth MTT. The MTT will exist for at least as long as the MOTS remains strictly-stably-outermost. This is a conceptually important result for numerical relativity because it shows that a large class of MOTSs behave regularly under time evolution. How is this to be reconciled with the known fact that $\mathrm{AHs}$ can jump during a time evolution? The reason is simply because of the outermost property. It is possible that a new MOTS can appear on the outside of a given MOTS. The "old" MOTS is then no longer the globally outermost one even though it is locally outermost, and it continues to evolve in a perfectly regular manner, but it is no longer an $\mathrm{AH}$.

There are, as yet, no similar existence proofs for MOTSs which are not strictly-stably-outermost. However, as we shall see later, we find in all the examples we have looked at, that MOTSs evolve smoothly even in this case, forming a regular world tube.

Isolated and dynamical horizons: A MTT is null in equilibrium situations when no matter or radiation is falling into it; the rest of the spacetime is still allowed to be highly dynamical. This situation is formalized by the notion of an isolated horizon [11-15]. Using isolated horizons, it has been possible to derive the laws of black hole mechanics, use it as a basis for the quantum black hole entropy calculations and find unexpected properties of hairy black holes in Einstein-Yang-Mills theory; see [19] and references therein. Most importantly for our purposes, isolated horizons have also proved to be useful in numerical relativity. For example, isolated horizons provide a coordinate invariant method of calculating the angular momentum and mass of a black hole [35]. They can be used to obtain boundary conditions for constructing quasiequilibrium initial data sets [36-39]. They might have a role in waveform extraction [15]. A pedagogical review of isolated horizons from the numerical relativity perspective can be found in [21].

In this paper, we are more interested in the dynamical regime when the MTT is not null. A spacelike MTT con- 
sisting of future-marginally trapped surfaces is called a dynamical horizon (DH). Thus, a dynamical horizon is a spacelike 3-surface equipped with a given foliation by FMOTSs. The properties of a dynamical horizon are studied in detail in $[9,10,40]$. The case when the horizon is very close to being isolated but still evolving dynamically has been studied in $[41,42]$ and its Hamiltonian treatment is considered in [43]. Note that the local existence of DHs follows from the local existence of MTTs because if $\Theta_{(n)}<0$ at any given time, it will continue to be strictly negative for at least a short duration. We elaborate on the spacelike property below.

A timelike MTT will be called a timelike membrane (TLM). A TLM cannot be considered to represent the surface of a black hole since a timelike surface is not a one-way membrane, and both ingoing and outgoing causal curves can pass through it. In some instances, we shall use the term "horizon" loosely to refer to a generic marginal surface or a MTT without any further qualifiers. The exact meaning should hopefully be clear from the context.

An explicit example of a dynamical horizon is provided by the Vaidya spacetime which describes the gravitational collapse of null dust [44-46]. (See also [47] for further examples in spherically symmetry). More generally, Fig. 1 depicts a dynamical horizon $H$ bounded by two MOTSs $S_{1}$ and $S_{2} . S$ is a typical member of the foliation. The vector $\hat{\tau}^{a}$ is the future directed unit timelike normal to $H, \hat{r}^{a}$ is tangent to $H$ and is the unit outward pointing spacelike normal to the cross sections. A fiducial set of null normals is

$$
\ell^{a}=\frac{1}{\sqrt{2}}\left(\hat{\tau}^{a}+\hat{r}^{a}\right), \quad n^{a}=\frac{1}{\sqrt{2}}\left(\hat{\tau}^{a}-\hat{r}^{a}\right) .
$$

As before, $\Theta_{(\ell)}=0$ and $\Theta_{(n)}<0$. The area of a cross section $S$ will be denoted by $A_{S}$ and its radius by $R_{S}:=$ $\sqrt{A_{S} / 4 \pi}$. A radial coordinate on $H$ will be denoted by $r$; the cross sections of $H$ are the constant $r$ surfaces. The 3metric and extrinsic curvature of $H$ will be denoted, respectively, by $q_{a b}$ and $K_{a b}$, and $\tilde{q}_{a b}$ is the 2-metric on $S$.

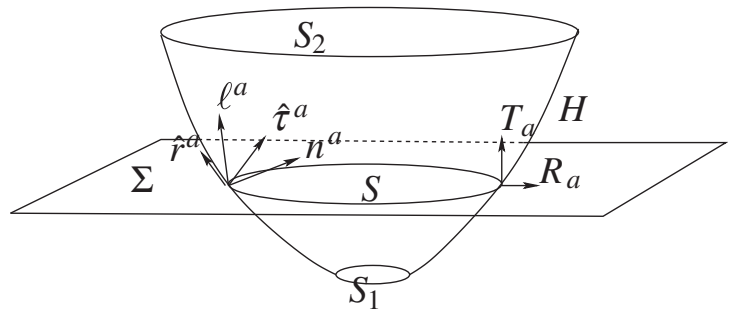

FIG. 1. A dynamical horizon $H$ bounded by MOTSs $S_{1}$ and $S_{2}$. $\ell^{a}$ is the outgoing null normal, $n^{a}$ is the ingoing null normal, $\hat{r}^{a}$ is the unit spacelike normal to the cross sections, and $\hat{\tau}^{a}$ is the unit timelike normal to $H$. $\Sigma$ is a Cauchy surface intersecting $H$ in a 2 -sphere $S . T^{a}$ is the unit timelike normal to $\Sigma$ and $R^{a}$ is the unit spacelike outward pointing vector normal to $S$ and tangent to $\Sigma$.
Figure 1 shows also a Cauchy surface $\Sigma$ intersecting a dynamical horizon $H$. This intersection $S$ will always be assumed to be one of the given cross sections of $H$. The unit timelike normal to the horizon is $T^{a}$ and the unit outward pointing spacelike normal to $S$ within $\Sigma$ is $R^{a}$. The three metric and extrinsic curvature of $\Sigma$ are denoted by $\bar{q}_{a b}$ and $\bar{K}_{a b}$ respectively. The fiducial set of null normals to $S$ arising naturally from $\Sigma$ are

$$
\bar{\ell}^{a}=\frac{1}{\sqrt{2}}\left(T^{a}+R^{a}\right), \quad \bar{n}^{a}=\frac{1}{\sqrt{2}}\left(T^{a}-R^{a}\right) .
$$

A boost transformation of the form of Eq. (2.2) connects $\left(\ell^{a}, n^{a}\right)$ and $\left(\bar{\ell}^{a}, \bar{n}^{a}\right)$ :

$$
\ell^{a}=f \bar{\ell}^{a}, \quad n^{a}=f^{-1} \bar{n}^{a} .
$$

When the horizon settles down and becomes null, an infinite boost $(f \rightarrow \infty)$ is required to go from $\left(\bar{\ell}^{a}, \bar{n}^{a}\right)$ to $\left(\ell^{a}, n^{a}\right)$.

We conclude this subsection with a short summary of some basic properties of a dynamical horizon:

Topology: The cross sections of a DH can be either spherical or toroidal $[9,10,16,34]$. Toroidal topology is possible only in exceptional cases when $\sigma_{(\ell) a b}$, the scalar curvature $\tilde{R}$ of $S, \mathcal{L}_{\ell} \Theta_{(\ell)}, R_{a b} \ell^{b}$, and $\zeta^{a}$ (defined in Sec. III) all vanish on $S$ [10]. We shall therefore always take the cross sections to be spherical. There are no similar results for cross sections of TLMs. However, we use an apparent horizon tracker which can only locate spherical AHs [48] and therefore all observed MOTSs have spherical topology.

Second Law: The area of the cross sections of a $\mathrm{DH}$ increases along $\hat{r}^{a}[9,10]$. Thus, if we choose a time evolution vector field $t^{a}$ for which $t \cdot \hat{r}>0$, then the area of the dynamical horizon will increase in time, and this result can be called the second law for dynamical horizons. Similarly, the area of a TLM decreases if $\Theta_{(n)}<0$, and increases if $\Theta_{(n)}>0$.

Foliation and Uniqueness: Any given spacelike MTT cannot have more than one distinct dynamical horizon structure on it [40]. This means that a DH can have one, and only one foliation by FMOTSs. This further implies that if a Cauchy surface $\Sigma$ does not intersect a given DH in one of the preferred cross sections, then the intersection cannot be a MOTS at all. Thus, different choices of Cauchy surfaces in general lead to different dynamical horizons. There are however some constraints on the location of dynamical horizons and trapped surfaces as proved by Ashtekar and Galloway [40]. For example, they show that given a dynamical horizon $H$ (along with a mild genericity assumption), there cannot be any trapped surfaces (and therefore no DHs) contained entirely in the past domain of dependence of $H$. See also $[46,49]$ for further discussion. 


\section{The signature of a MTT}

As discussed above, MTTs have been shown to exist for a large and physically interesting class of MOTSs, and this is borne out in a large number of numerical simulations where MOTSs are located and evolved smoothly. How many of these MTTs are actually dynamical horizons? In other words, when is a MTT spacelike? The first result in this direction was obtained by Hayward [16] (see also [35]). Using the Raychaudhuri equation for $\ell^{a}$, it can be shown that a MTT is spacelike if $\alpha<0$, null if $\alpha=0$ and timelike if $\alpha>0$, where

$$
\alpha \equiv \frac{\sigma_{(\ell) a b} \sigma_{(\ell)}^{a b}+R_{a b} \ell^{a} \ell^{b}}{\mathcal{L}_{n} \Theta_{(\ell)}} .
$$

In writing this expression, it is assumed that $\ell^{a}$ and $n^{a}$ are extended off $H$ geodetically, so that $\mathcal{L}_{n} \Theta_{(\ell)}$ is meaningful. The term in the numerator is strictly positive in the case of dynamical horizons if the matter fields satisfy, say, the null energy condition. It vanishes for isolated horizons. The denominator is negative for the Vaidya spacetime and also for the stationary Kerr-Newman family. This captures the notion that as we go inside the black hole, the outgoing null rays become more and more converging. Assuming that the numerator of Eq. (2.10) is nowhere vanishing on $H$, the hypothesis that $H$ is spacelike is equivalent to $\mathcal{L}_{n} \Theta_{(\ell)}<0$. As shown by Ben-Dov [50], this last condition is not satisfied for all MTTs; in Oppenheimer-Snyder collapse [51], there exists a timelike world tube of FMOTSs with $\mathcal{L}_{n} \Theta_{(\ell)}>0$.

The issue of the signature has been considered in [34]. There it is shown that if a MOTS $S$ is strictly-stablyoutermost, and if the quantity $\sigma_{(\ell) a b} \sigma_{(\ell)}^{a b}+R_{a b} \ell^{a} \ell^{b}$ is nonzero somewhere on $S$ (and assuming the null energy condition), then the MTT containing $S$ is spacelike in a neighborhood of $S$. This result is stronger than Hayward's result [Eq. (2.10)] and it shows clearly that the spacelike case is physically the most interesting because $\sigma_{(\ell) a b} \sigma_{(\ell)}^{a b}+R_{a b} \ell^{a} \ell^{b}$ will not vanish in a nonstationary situation. It also shows, somewhat surprisingly, that even if matter or radiation is falling into a black hole only in the form of say, a single narrow beam from a particular direction, the entire MTT is spacelike. One might naively have thought that the MTT would be spacelike only on portions where the energy flux is nonzero, and null otherwise. This is not the case because of the elliptic nature of the equations governing the deformations of a MOTS. ${ }^{3}$

In all the examples we present later, it turns out that MOTSs form in pairs, i.e., just after a MOTS $S_{0}$ appears

\footnotetext{
${ }^{3}$ The Oppenheimer-Snyder case studied in [50] does not satisfy the hypotheses of these theorems because for this case, the matter fields have a discontinuity at the surface of the star. Further examples in spherical symmetry are studied in [47] where the matter fields are smooth.
}

initially, it bifurcates into "outer" and "inner" MTTs, $H_{\text {out }}$ and $H_{\text {in }}$, respectively. The initial MOTS $S_{0}$ is the common cross section of $H_{\text {out }}$ and $H_{\mathrm{in}}$, and the union $H_{\text {tot }}=$ $H_{\text {out }} \bigcup H_{\text {in }}$ forms a single smooth manifold, as far as we can tell numerically (though a more detailed analysis of the differentiability of $H_{\text {tot }}$ is required). In particular, the area of the cross sections is a differentiable and monotonic function on this manifold. Furthermore, $H_{\text {out }}$ is spacelike, even on the initial cross section $S_{0}$. This means that the inner MTT $H_{\text {in }}$ is, by continuity, initially spacelike in an open neighborhood of $S_{0}$. However, in some cases $H_{\text {in }}$ soon acquires a mixed signature and becomes more and more timelike, and ends up as a TLM. We strongly suspect that such a bifurcation is a general phenomenon whenever a new MOTS is formed. The MOTSs on the inner MTT are not strictly-stably-outermost and thus $H_{\text {in }}$ is not required to be spacelike according to the results of [34].

There is one configuration where the existence of an inner MTT is plausible. Figure 2 shows two MOTSs $S_{(1),(2)}$ surrounded by a common MOTS $S_{\text {out }} ; \Theta_{(\ell)}$ vanishes on all these surfaces. Let us assume that $S_{(1)}, S_{(2)}$, and $S_{\text {out }}$ are all strictly-stably-outermost and that deforming $S_{(1)}$ and $S_{(2)}$ outward yields strictly untrapped surfaces $S_{(1)}^{\prime}$ and $S_{(2)}^{\prime}$. Similarly, suppose that deforming $S_{\text {out }}$ inwards gives a strictly trapped surface $S_{\text {out }}^{\prime}$. Then, since $\Theta_{(\ell)}$ must change sign somewhere between $S_{\text {out }}^{\prime}$ and $S_{(1)}^{\prime}$ or $S_{(2)}^{\prime}$, it is plausible that there is a MOTS $S_{\text {in }}$ in the intermediate region inside $S_{\text {out }}$ and outside $S_{1}$ and $S_{2}$. This argument is supported by a recent result by Schoen [52] which shows the existence of a MOTS between a trapped (in our case $S_{\text {out }}^{\prime}$ ) and an untrapped surface (in our case $S_{(1)}^{\prime} \bigcup S_{(2)}^{\prime}$ ). It might be possible to extend this proof to rigorously prove the existence of $S_{\text {in }}$ in our case, and to check whether it is topologically a sphere. $S_{(1)}, S_{(2)}$, and $S_{\text {out }}$ are cross sections of a dynamical horizon while $S_{\text {in }}$ is a cross section of a MTT, not necessarily a dynamical horizon.

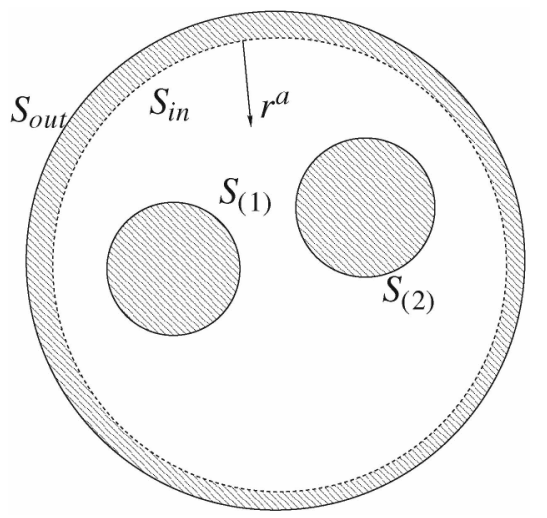

FIG. 2. Two MOTSs $S_{(1)}$ and $S_{(2)}$ surrounded by a common MOTS $S_{\text {out }}$. Spheres lying just inside these FMOTSs must have negative outgoing expansion. Thus, there must be a inner trapped horizon $S_{\text {in }}$ inside $S_{\text {out }}$ which encloses $S_{(1)}$ and $S_{(2)}$. 


\section{APPLICATIONS}

This section discusses some possible applications of dynamical horizons. These ideas are illustrated using concrete numerical examples later in Sec. IV.

\section{A. Computing the signature of a MTT}

From a numerical standpoint, it is more convenient to deduce the signature of $H$ by directly calculating the induced metric $q_{a b}$, rather than from Eq. (2.10) by calculating $\mathcal{L}_{n} \Theta_{(\ell)}$ which requires extensions of $\ell^{a}$ and $n^{a}$ away from the horizon. The signature of $H$ is then determined by the sign of the determinant of $q_{a b}$ which is gauge independent; note that the determinant is itself gauge dependent. To calculate $q_{a b}$ we find a frame $\mathbf{e}_{(i)}^{a}(i=1,2,3)$ on $H$, i.e., three smooth vector fields on $H$ which are pointwise linearly independent. We then simply need to compute the determinant of the matrix

$$
\mathbf{q}_{(i)(j)}:=g_{a b} \mathbf{e}_{(i)}^{a} \mathbf{e}_{(j)}^{a} .
$$

We construct a frame on $H$ as follows. Let $\left(t, x^{i}\right)(i=$ $1,2,3)$ be the spacetime coordinates on $\mathcal{M}$ used in the numerical simulation. The MTT $H$ is topologically $I \times S^{2}$ $(I$ some interval in $\mathbb{R})$ so that we can assume coordinates $(r, \theta, \phi)$ on it. Here $(\theta, \phi)$ are standard coordinates on $S^{2}$ and $r$ is a radial coordinate. We can use the time coordinate $t$ as the radial coordinate $r$ on $H$ by considering $H$ to be embedded into the spacetime $\mathcal{M}$ by means of the map

$$
F(r, \theta, \phi)=\left(t=r, x^{i}=F^{i}(r, \theta, \phi)\right) .
$$

The maps $F^{i}$ are known as soon as the MOTSs are found by the AH tracker. As a frame on $H$ we choose

$$
\mathbf{e}_{(1)}=\partial_{\theta}, \quad \mathbf{e}_{(2)}=\frac{1}{\sin \theta} \partial_{\phi}, \quad \mathbf{e}_{(3)}=\partial_{r} .
$$

Hence, $\mathbf{e}_{(3)}$ connects a point on a MOTS at a certain instant of time with a corresponding point on the MOTS at the next instant of time. Note that this choice of frame breaks down at the poles of the sphere. To apply formula (3.1), the frame (3.3) on $H$ must be pushed forward to $\mathcal{M}$ by means of the embedding $F$ in the standard way:

$$
\mathbf{e}_{(3)}=\left(1, \partial_{r} F^{1}, \partial_{r} F^{2}, \partial_{r} F^{3}\right) .
$$

This enables us to calculate $\mathbf{q}_{(i)(j)}$ using the 3-metric on the Cauchy surface, and the lapse and shift.

Having calculated the matrix $\mathbf{q}_{(i)(j)}$ and assuming its determinant to be positive, we can easily calculate the unit vector $\hat{r}^{a}$. It is simply the outward pointing unit spacelike vector which is a linear combination of $\left(\mathbf{e}_{(1)}, \mathbf{e}_{(2)}, \mathbf{e}_{(3)}\right)$, and is orthogonal to $\mathbf{e}_{(1)}$ and $\mathbf{e}_{(2)}$. This construction of $\hat{r}^{a}$ will also work in the timelike case, but not in the null case where $\mathbf{q}_{(i)(j)}$ becomes degenerate.

\section{B. Angular momentum and mass}

Let $\varphi^{a}$ be a rotational vector field on $H$ tangent to each cross section. ${ }^{4}$ The angular momentum of a cross section $S$ associated with $\varphi^{a}$ is given by

$$
J_{S}^{(\varphi)}=-\frac{1}{8 \pi} \oint_{S} K_{a b} \varphi^{a} \hat{r}^{b} d^{2} V .
$$

We refer to [10] for a justification for this formula. The interpretation of $J_{S}^{(\varphi)}$ as angular momentum is most clear cut when $\varphi^{a}$ is a rotational symmetry on $H$, i.e., when $\mathcal{L}_{\varphi} K_{a b}=0$ and $\mathcal{L}_{\varphi} q_{a b}=0$. See [35] for a method of finding Killing vectors suitable for numerical implementation. Booth and Fairhurst have shown that this formula also arises from a Hamiltonian calculation [43]. As we shall see below, $J_{S}^{(\varphi)}$ is also gauge invariant when $\varphi^{a}$ is only divergence free, and not necessarily a symmetry vector. However, $J_{S}^{(\varphi)}$ may not be meaningful for more general $\varphi^{a}$.

If a cross section $S$ has radius $R_{S}$ and angular momentum $J_{S}^{(\varphi)}$, we can meaningfully talk about the mass:

$$
M_{S}^{(\varphi)}=\frac{1}{2 R_{S}} \sqrt{R_{S}^{4}+4\left(J_{S}^{(\varphi)}\right)^{2}} .
$$

This mass has the same dependence on the area and angular momentum as in the Kerr solution. There is a meaningful balance law for the mass and furthermore, it satisfies a physical process version of the first law $[9,10]$.

Equation (3.5) uses the metric $q_{a b}$ and $K_{a b}$ and extrinsic curvature of the dynamical horizon. It is more convenient to recast this in terms of the metric $\bar{q}_{a b}$ and extrinsic curvature $\bar{K}_{a b}$ of the partial Cauchy surface $\Sigma$ (see Fig. 1). It is also more convenient to work with the null normals $\left(\bar{\ell}^{a}, \bar{n}^{a}\right)$ defined in Eq. (2.8). It is clear that $\left(\bar{\ell}^{a}, \bar{n}^{a}\right)$ must be related to the old null normals $\left(\ell^{a}, n^{a}\right)$ by a boost transformation, i.e., there must exist a positive function $f$ on $S$ such that

$$
\ell^{a}=f \bar{\ell}^{a} \quad \text { and } \quad n^{a}=f^{-1} \bar{n}^{a} .
$$

After some simple algebra, Eq. (3.5) can be written as:

$$
J_{S}^{(\varphi)}=-\frac{1}{8 \pi}\left(\oint_{S} \bar{K}_{a b} R^{a} \varphi^{b} d^{2} V+\oint_{S} \mathcal{L}_{\varphi} \ln f d^{2} V\right) .
$$

The second integral vanishes precisely when $\varphi^{a}$ is divergence free, i.e., when $\varphi^{a}$ is a symmetry of the area element on $S$. In this case:

$$
J_{S}^{(\varphi)}=-\frac{1}{8 \pi} \oint_{S} \bar{K}_{a b} R^{a} \varphi^{b} d^{2} V .
$$

In particular, this will be true when $\varphi^{a}$ is a symmetry of the metric $\tilde{q}_{a b}$, but the divergence free condition is much weaker than this. For example, following [19], we can

\footnotetext{
${ }^{4}$ This means that $\varphi^{a}$ is tangent to $S$, has closed integral curves, and is normalized so that its integral curves have an affine length of $2 \pi$, and it vanishes at exactly two points on $S$.
} 
always construct a divergence free vector field on a 2sphere even in the absence of axisymmetry as follows. Let $h$ be any smooth function on $S$, and $g$ another smooth function satisfying $\tilde{\epsilon}^{a b} \partial_{a} h \partial_{b} g=0$, where $\tilde{\epsilon}_{a b}$ is the volume form on $S$. It is easy to check explicitly that the following vector field is automatically divergence free:

$$
\tilde{\varphi}^{a}=g \tilde{\epsilon}^{a b} \partial_{b} h .
$$

The integral curves of $\tilde{\varphi}^{a}$ are the level curves of $h$. In particular, if $h$ is chosen to be a geometric quantity such as, say, the curvature $\tilde{R}$, and $g$ chosen such that $\tilde{\varphi}^{a}$ has affine length $2 \pi$, then $\tilde{\varphi}^{a}$ will coincide with an axial Killing vector, if it exists. Therefore, $\tilde{\varphi}^{a}$ can be viewed as an ersatz axial symmetry vector field even in the absence of axisymmetry.

However, we have not as yet satisfactorily implemented the above construction due to numerical difficulties arising from errors in taking derivatives of the scalar curvature. Furthermore, the $\varphi^{a}$ coming from Eq. (3.9) may not look like a rotational vector field;, in particular, it may vanish at more than just two points on the sphere even when $S$ is close to axisymmetry. ${ }^{5}$ This is work in progress. The results presented below all use the method described in [35] of finding Killing vectors based on the Killing transport equations. This reduces the problem of finding Killing vectors on a sphere to the diagonalization of a $3 \times 3$ matrix, and integrating a 1-dimensional ordinary differential equation. We have found this method to be quite reliable for the cases when the horizon is sufficiently close to axisymmetry, even in cases when the coordinate system is not adapted to the axial symmetry. Thus, it works well for the head-on collision and axisymmetric neutron star collapse, but only at very early and late times for a nonaxisymmetric black hole collision. This caveat only affects the example of Sec. IV B. It is important to keep in mind that this Killing transport method is not reliable for checking whether the horizon is close to axisymmetry; this requires an independent calculation of $\mathcal{L}_{\varphi} \tilde{q}_{a b}$ to verify that it is sufficiently small. Finally, we emphasize that this method is also not guaranteed to produce a divergence free rotational vector field; this must also be checked independently.

\section{Multipole moments}

The notion of multipole moments play a very important role in Newtonian gravity and classical electrodynamics. Let us focus on classical electrodynamics in Minkowski space with axisymmetric charge and current distributions $\rho$ and $j_{a}$ respectively, given on a sphere $S$ of radius $R_{S}$. Let $(\theta, \phi)$ be coordinates on $S ; \rho$ and $j_{a}$, being axisymmetric, are functions only of $\theta$. The electric multipoles $E_{n}$ and magnetic multipoles $B_{n}$ are, respectively, defined as

\footnotetext{
${ }^{5}$ We thank Ivan Booth for this comment.
}

$$
\begin{gathered}
E_{n}=R_{S}^{n} \oint \rho P_{n}(\cos \theta) d^{2} V, \\
B_{n}=-R_{S}^{n+1} \oint_{S}\left(\vec{j} \times \overrightarrow{\tilde{\partial}} P_{n}(\cos \theta)\right) \cdot \hat{n} d^{2} V,
\end{gathered}
$$

where $P_{n}$ is the $n$th Legendre polynomial, $\tilde{\partial}$ denotes the standard derivative operator on a sphere, and $\hat{n}$ is the unit outward normal to the sphere. For black holes, the analogs of the electric and magnetic multipole moments are, respectively, the mass and angular momentum multipole moments. Motivated by this analogy, there exist meaningful definitions of the source multipole moments for an isolated horizon [53]. Roughly speaking, these definitions correspond to taking the moments of the free data on an axisymmetric isolated horizon, and knowledge of these moments is sufficient to construct the entire horizon geometry.

For dynamical horizons, we can generalize the construction of [53] to construct a set of multipole moments which capture the geometry of a dynamical horizon at any instant of time, and which are furthermore equal to the isolated horizon multipole moments when the black hole is isolated. The analog of charge density is (proportional to) the scalar curvature on $S$ :

$$
\rho_{S}=\frac{1}{8 \pi} M_{S} \tilde{\mathcal{R}}
$$

and the angular momentum current is

$$
j_{a}=-\frac{1}{8 \pi} \tilde{q}_{a}^{c} \bar{K}_{c b} R^{b} .
$$

The moments of these quantities will give the desired multipole moments. We could also use $\tilde{q}_{a}^{c} K_{c b} \hat{r}^{b}$ instead of $\tilde{q}_{a}^{c} \bar{K}_{c b} R^{b}$ above; the two expressions are related by a boost transformation. Just as for angular momentum, the final expressions for the multipole moments given below will be boost invariant if the $\varphi^{a}$ used in their definition is divergence free. To define the moments, we need a preferred coordinate system on $S$ so that we can define the preferred spherical harmonics.

The construction of the preferred coordinate system $(\theta, \phi)$ on $S$ is the same as given in [53]: $\phi \in[0,2 \pi)$ is the affine parameter along $\varphi^{a}$ and $\zeta:=\cos \theta \in[-1,1]$ is defined by the condition

$$
\tilde{D}_{a} \zeta=\frac{1}{R_{S}^{2}} \tilde{\epsilon}_{b a} \varphi^{a} .
$$

The freedom to add a constant to $\zeta$ is removed by requiring its integral over $S$ to vanish: $\oint_{S} \zeta d^{2} V=0$. When applied to a Kerr black hole, these invariant coordinates turn out to be the same as the usual Boyer-Lindquist $(\theta, \phi)$ coordinates.

The mass and angular multipole moments are then respectively: 


$$
\begin{gathered}
M_{n}=\frac{R_{S}^{n} M_{S}}{8 \pi} \oint_{S}\left\{\tilde{\mathcal{R}} P_{n}(\zeta)\right\} d^{2} V, \\
J_{n}=-\frac{R_{S}^{n+1}}{8 \pi} \oint_{S}\left\{\tilde{\epsilon}^{a b}\left(\partial_{b} P_{n}(\zeta)\right) K_{a c} R^{c}\right\} d^{2} V \\
=\frac{R_{S}^{n-1}}{8 \pi} \oint_{S} P_{n}^{\prime}(\zeta) \bar{K}_{a b} \varphi^{a} R^{b} d^{2} V
\end{gathered}
$$

where $P_{n}^{\prime}(\zeta)=d P_{n}(\zeta) / d \zeta$. We have used Eq. (3.14) to obtain the final expression for $J_{n}$ above. This form clarifies the relation of $J_{n}$ to the angular momentum and also demonstrates the gauge invariance of $J_{n}$ when $\varphi^{a}$ is divergence free. Using the Gauss-Bonnet theorem, it is trivial to check that $M_{0}=M_{S}$ and $J_{1}=J_{S} . J_{0}$ vanishes because we do not consider any topological defects. Furthermore, these expressions are well suited for numerical computation because they involve only quantities on the Cauchy surface and an integral over the MOTS.

\section{The energy and angular momentum fluxes}

Hawking's area theorem shows that if matter satisfies the null energy condition, then the area of the event horizon can never decrease. This is one of the central results of black hole physics, and it leads to the classical picture of the black hole growing inexorably as it swallows matter and radiation. Therefore, one might expect there to be a balance law relating the increase in area to fluxes of matter and radiation crossing the event horizon. However, the teleological nature of event horizons is again a problem; there cannot exist any such local balance law for the area of the event horizon. A clear example is seen in the Vaidya spacetime where the event horizon is formed in flat space and its area increases in anticipation of matter falling into the black hole at a later time; see [19] for a discussion.

For DHs, it is possible to obtain an exact balance law for the area increase $[9,10]$; i.e., given two cross sections $S_{1}$ and $S_{2}$ with radii $R_{1}$ and $R_{2}$ respectively, and with $S_{2}$ lying to the outside of $S_{1}$, the increase in the radius is given by the sum of the energy flux due to matter $\left(\mathcal{F}^{(m)}\right)$ and gravitational radiation $\left(\mathcal{F}^{(g)}\right)$, both of which are manifestly positive:

$$
\frac{R_{2}-R_{1}}{2}=\mathcal{F}^{(m)}+\mathcal{F}^{(g)},
$$

where

$$
\begin{array}{r}
\mathcal{F}^{(m)}=\int_{H} \sqrt{2} T_{a b} \hat{\tau}^{a} \ell^{b} d R d^{2} V, \\
\mathcal{F}^{(g)}=\frac{1}{8 \pi} \int_{H}\left\{\left|\sigma_{(\ell)}\right|^{2}+|\zeta|^{2}\right\} d R d^{2} V .
\end{array}
$$

Here $\left|\sigma_{(\ell)}\right|^{2}:=\sigma_{(\ell) a b} \sigma_{(\ell)}^{a b},|\zeta|^{2}:=\zeta_{a} \zeta^{a}$ where $\zeta^{a}$ is a vector on $S$ defined as

$$
\zeta^{a}:=\sqrt{2} \tilde{q}^{a b} \hat{r}^{c} \nabla_{c} \ell_{b},
$$

and $d^{2} V$ is the natural geometric volume element on $H$. The extra factors of 2 and $\sqrt{2}$ in the above equations as compared to the corresponding equations in [10], arise because of our normalization convention $\ell \cdot n=-1$; [10] uses $\ell \cdot n=-2$.

See [10] for additional reasons why $\mathcal{F}^{(g)}$ has the right properties to be viewed as the flux of gravitational radiation. Equation (3.17) is an exact statement about black holes in full nonlinear general relativity, and it is the analog of the Bondi mass balance law at null infinity.

From a numerical point of view, $\mathcal{F}^{(g)}$ is inconvenient to calculate, especially when the horizon is settling down and is close to being null. First of all, we have direct access only to the fiducial null normals $\left(\bar{\ell}^{a}, \bar{n}^{a}\right)$ defined in Eq. (2.8) and not to $\left(\ell^{a}, n^{a}\right)$ themselves. The two sets of null normals are related to each other by a boost transformation $\ell^{a}=f \bar{\ell}^{a}, n=f^{-1} \bar{n}^{a}$. Under this transformation, $\sigma_{\ell}=f \sigma_{\bar{\ell}}$. Similarly, it is easy to show that

$$
\zeta^{a}=f^{2} \bar{\kappa}^{a}-\bar{\omega}^{a},
$$

where

$$
\bar{\kappa}^{a}=\tilde{q}^{a b} \bar{\ell}^{c} \nabla_{c} \bar{\ell}_{b} \quad \text { and } \quad \bar{\omega}^{a}=\tilde{q}^{a b} \bar{n}^{c} \nabla_{c} \bar{\ell}_{b} .
$$

Here $\bar{\kappa}^{a}$ and $\bar{\omega}^{a}$ are tangent to the cross sections of the DH. When the DH approaches equilibrium, $f \rightarrow \infty$. However, the value of $\mathcal{F}^{(g)}$ itself remains finite. All fields with a bar remain finite even when the horizon becomes null even though $f$ diverges. While this is not a problem analytically, this does cause numerical errors in the transition to equilibrium when we multiply a very small quantity on the horizon with a very large one. This is consistent with the results of [41] where it is found that $\left|\sigma_{(\bar{\ell})}\right|^{2}$ is the most important when the horizon is close to equilibrium.

Let $t$ be the time coordinate used to label the Cauchy surfaces. Using this coordinate, we can identify the divergence of various terms appearing in $\mathcal{F}^{(g)}$. We start by rewriting $\mathcal{F}^{(g)}$ as:

$$
\mathcal{F}^{(g)}=\frac{1}{8 \pi} \int_{H}\left\{\left|\sigma_{(\ell)}\right|^{2}+|\zeta|^{2}\right\} \frac{d R}{d t} d^{2} V d t .
$$

The integrand on the right hand side can be expanded as

$$
\begin{aligned}
\left(\left|\sigma_{(\ell)}\right|^{2}+|\zeta|^{2}\right) \dot{R}= & \dot{R} f^{4}|\bar{\kappa}|^{2}+\dot{R} f^{2}\left(\left|\sigma_{(\bar{\ell})}\right|^{2}-\bar{\omega} \cdot \bar{\kappa}\right) \\
& +\dot{R}|\bar{\omega}|^{2} .
\end{aligned}
$$

Let us look at the various terms in this expression. First, $\bar{\omega}^{a}$ can be shown to be equal to the angular momentum current; for an axial symmetry vector $\varphi^{a}$, the angular momentum is simply the integral of $\varphi^{a} \bar{\omega}_{a}$ over the cross section of the MTT. Thus, $\bar{\omega}_{a}$ need not vanish even when the MTT becomes an isolated horizon. The $|\bar{\omega}|^{2}$ term in the flux can, in some sense, be viewed as the flux of rotational energy entering the horizon. Now consider $\bar{\kappa}^{a}$. For an isolated horizon, $\bar{\ell}^{b} \nabla_{b} \bar{\ell}^{a} \propto \bar{\ell}^{a}$ because in this case $\bar{\ell}^{a}$ is guaranteed 
to be geodetic. This implies $\bar{\kappa}^{a}=0$. On the dynamical horizon side, we can choose suitable extensions of $\bar{\ell}^{a}$ (and $\bar{n}^{a}$ ) away from the MTT so that $\bar{\kappa}^{a}=0$. The shear $\sigma_{(\bar{\ell})}$ on the other hand contains most of the nontrivial information about the radiation falling into the black hole. It vanishes on an isolated horizon as it should, and it is independent of any extensions of $\bar{\ell}^{a}, \bar{n}^{a}$ away from the MTT. Therefore, in the examples of Sec. IV, we shall usually plot $\sigma_{(\bar{\ell})}$ to show the energy flux falling into the horizon.

The angular momentum also obeys a balance law similar to Eq. (3.17):

$$
J_{2}-J_{1}=\mathcal{J}_{\varphi}^{(m)}+\mathcal{J}_{\varphi}^{(g)}
$$

where

$$
\begin{array}{r}
\mathcal{J}_{\varphi}^{(m)}=-\int_{\Delta H} T_{a b} \hat{\tau}^{a} \varphi^{b} d^{3} V, \\
\mathcal{J}_{\varphi}^{(g)}=-\frac{1}{16 \pi} \int_{\Delta H} P^{a b} \mathcal{L}_{\varphi} q_{a b} d^{3} V
\end{array}
$$

where $P^{a b}:=K^{a b}-K q^{a b}$. Unlike the energy flux $\mathcal{F}^{(g)}$, the angular momentum flux $\mathcal{J}^{(g)}$ is not positive definite. Also, $\mathcal{J}^{(g)}$ vanishes when $\varphi^{a}$ is an axial Killing vector on $H$. Thus, angular momentum is conserved in the axisymmetric vacuum case, as it should be.

\section{EXAMPLE NUMERICAL SIMULATIONS}

In this section, we apply the ideas discussed in the previous sections to three concrete numerical simulations: (i) A head-on collision of two black holes starting with Brill-Lindquist initial data; (ii) A nonaxisymmetric black hole collision using puncture initial data with nonvanishing linear momentum; and (iii) Axisymmetric collapse of a neutron star. Each of these three cases is quite well known in the numerical relativity literature, and all have been well studied. This section aims to further explore these examples using the tools described in Sec. III.

\section{A. Head-on collision with Brill-Lindquist data}

The Brill-Lindquist initial data [54] for binary black holes represent initial data for two nonspinning black holes without any orbital angular momentum. The reader can consult a review on initial data, such as [55] for details. Here we simply note that these initial data are conformally flat and time-symmetric:

$$
\bar{q}_{a b}=\psi^{4} \delta_{a b}, \quad \bar{K}_{a b}=0 .
$$

The manifold $\Sigma$ is $\mathbb{R}^{3}$ with two points removed (the punctures). The only equation to be solved is the flat space Laplace equation for the conformal factor:

$$
\Delta \psi=0 .
$$

Let $d$ denote the shortest distance between the two punc- tures as measured with respect to the fictitious flat background metric $\delta_{a b}$; the physical proper distance between the punctures is actually infinite. It was shown in [54] that each of the punctures is actually an asymptotically flat region. The total ADM mass of the common asymptotic region is

$$
m_{\mathrm{ADM}}=2 \alpha_{(1)}+2 \alpha_{(2)},
$$

and the ADM masses of the two punctures are

$$
\begin{aligned}
& m_{(1)}^{\mathrm{ADM}}=2 \alpha_{(1)}+\frac{2 \alpha_{(1)} \alpha_{(2)}}{d} \\
& m_{(2)}^{\mathrm{ADM}}=2 \alpha_{(2)}+\frac{2 \alpha_{(1)} \alpha_{(2)}}{d} .
\end{aligned}
$$

These are exact results, irrespective of the distance $d$ between the punctures. In the next two subsections, we look at two different regimes (i) the far limit when $d$ is large and (ii) the merger of the two holes starting from relatively small values of $d$.

\section{The far limit}

Before presenting the results from the numerical evolution of this data, it is instructive to look at a special case which is amenable to analytic treatment, namely, in the far limit where the separation between the holes is very large: $d \gg \alpha_{(1)}, \alpha_{(2)}$. In this case, there are two MOTSs surrounding each of the punctures without any common MOTS surrounding them. The angular momenta of the two black holes are trivially zero because the extrinsic curvature vanishes. What about the mass? Should $m_{(1)}^{\mathrm{ADM}}$ and $m_{(2)}^{\mathrm{ADM}}$ be identified with the masses of the black holes? There are three difficulties with this. First, these ADM masses also include contributions from radiation present in the respective asymptotic regions. Secondly, if this identification is correct, $m_{(i)}^{\mathrm{ADM}}(i=1,2)$ is supposed to be the mass of the black hole for all values of $d$, even when the two black holes are very close to each other. Should not the mass of the black holes in this regime also include, say, contributions from the tidal distortions produced by the other hole? Finally, the strategy of using the asymptotic regions to define black hole masses is not applicable generally, say in the case when there are matter fields and the topology of $\Sigma$ is just $\mathbb{R}^{3}$, or in Misner data [56] where the two black holes do not have their own individual asymptotic regions.

From the isolated/dynamical horizon perspective, since the black holes have zero angular momentum, from Eq. (3.8), the irreducible mass is the correct measure of mass in this case: $m_{(i)}=\sqrt{a_{(i)} / 16 \pi}$ where $a_{(i)}$ is the area of the MOTS around each of the punctures. Let us then calculate the mass of the black holes as a power series in $1 / d$. To simplify calculations, put the origin of coordinates at the location of the first puncture and the other puncture 
on the $z$-axis at $(0,0, d)$. Introduce the usual spherical coordinates $(r, \theta, \phi)$ so that the conformal factor becomes explicitly

$$
\phi(r, \theta)=1+\frac{\alpha_{(1)}}{r}+\frac{\alpha_{(2)}}{r}\left(1-\frac{2 d \cos \theta}{r}+\frac{d^{2}}{r^{2}}\right)^{-1 / 2} .
$$

We see that due to axisymmetry, there is no dependence on $\phi$. Let the surface of the FMOTS around the origin be given by the equation $r=h(\theta)$. In the limit when $d \rightarrow \infty$, the initial data reduces to Schwarzschild in isotropic coordinates so that the horizon is located at $r=\alpha_{(1)}$.

Higher order effects can also be explicitly calculated. It turns out [57] that up to $\mathcal{O}\left(d^{-3}\right)$, the location of the MOTS is given by

$$
\begin{aligned}
r= & \alpha_{(1)}-\frac{\alpha_{(1)} \alpha_{(2)}}{d}+\frac{\alpha_{(1)} \alpha_{(2)}}{d}\left(\alpha_{(2)}-\alpha_{(1)} \cos \theta\right) \\
& -\frac{\alpha_{(1)} \alpha_{(2)}}{3}\left(\alpha_{(2)}^{2}-3 \alpha_{(1)} \alpha_{(2)} \cos \theta\right. \\
& \left.+\frac{5}{7} \alpha_{(1)}^{2} P_{2}(\cos \theta)\right)+\mathcal{O}\left(d^{-4}\right)
\end{aligned}
$$

where $P_{2}$ is the second Legendre polynomial. Using this result, the horizon mass $m_{(i)}=\sqrt{a_{(i)} / 16 \pi}$ can be calculated and, somewhat surprisingly, the mass is the same as the ADM mass even up to third order:

$$
m_{(1)}=2 \alpha_{(1)}+\frac{2 \alpha_{(1)} \alpha_{(2)}}{d}+\mathcal{O}\left(d^{-4}\right) .
$$

This relation was verified numerically for a sequence of BL data with different values of $d$. However, we did not have sufficient resolution to estimate the leading order deviation between $m_{(1)}$ and $m_{(1)}^{\mathrm{ADM}}$. Similarly, the shear of the horizon vanishes up to third order indicating that the individual horizons are isolated to an excellent approximation. As we shall see below, the individual horizons are isolated even for relatively small values of $d$ once the common MOTS has formed.

\section{Numerical results for the merger phase}

We performed a numerical evolution starting with BrillLindquist initial data. Working in units where the total ADM mass is unity, the punctures were located at $z=$ \pm 0.5 , and the individual black holes had equal masses. Thus $2 \alpha_{(1)}=2 \alpha_{(2)}=0.5$. The domain had an explicit octant symmetry and extended up to $x, y, z=96$. Near the outer boundary the spatial resolution was $h=1.6$, and near the punctures we used mesh refinement to increase the resolution successively up to $h=0.0125$, so that the individual horizon diameters contained initially 32 grid points. We used fourth order accurate spatial differencing operators, and a third order Runge-Kutta time integrator.

We excised [58] coordinate spheres with a radius of $r_{e}=$ 0.0625 about the punctures from the domain, correspond- ing to a diameter of 10 grid points. We used the AEI BSSN formulation $[58,59]$ for time evolution, using the boundary conditions also described in [58]. These boundary conditions are known to be incompatible with the Einstein equations. We used a $1+\log$ slicing condition [60] starting from $\alpha=1$, and a zero shift. This makes both the individual and the outer common horizon grow in coordinate space. We used the CACTUS framework $[61,62]$, the Carpet mesh refinement driver [63,64], and the CACTUSEINSTEIN infrastructure. We located the apparent horizon surfaces with J. Thornburg's AHFINDERDIRECT $[48,65]$.

In this setup, the apparent horizon has two disconnected components in the initial data, and a common MOTS forms shortly after $t=0.5$. The individual horizons are null up to numerical errors (consistent with the result on the smallness of $\sigma_{(\ell)}$ in the far limit), and their masses are essentially constant up to numerical error. As discussed in Sec. II B 2 and Fig. 2, the common MOTS forms initially as a single surface but then bifurcates: an outer horizon which is strictly-stably-outermost, and an inner one which becomes strictly untrapped on being deformed inwards. Figure 3 shows the shapes of the individual and the inner and outer common MOTSs at time $t=1$, where the inner and outer common MTTs have already noticeably separated. As expected, the outer MTT is purely spacelike while the inner MTT, being spacelike initially, becomes partly timelike quickly. Figure 4 shows the horizon world tube metric signature at $t=0.6$ and $t=1$. At later times, the outer MTT tends to become null (as expected), while the inner MTT becomes completely timelike, and then becomes so distorted at about $t=1.2$ that it cannot be reliably tracked any more. This coordinate distortion is already evident in Fig. 3, and the horizon discretization

\section{Horizon shapes at $\mathrm{t}=1$}

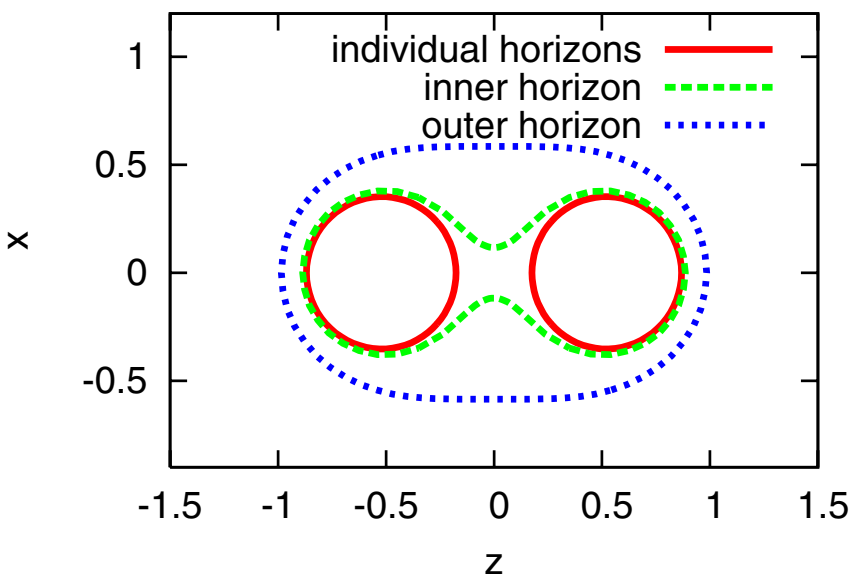

FIG. 3 (color online). Coordinate shapes of the horizons at $t=$ 1 in the $x z$ plane. A common horizon has formed, and the inner and outer common horizons have already separated. Compare Fig. 2. 
Horizon metric determinant at $\mathrm{t}=0.6$

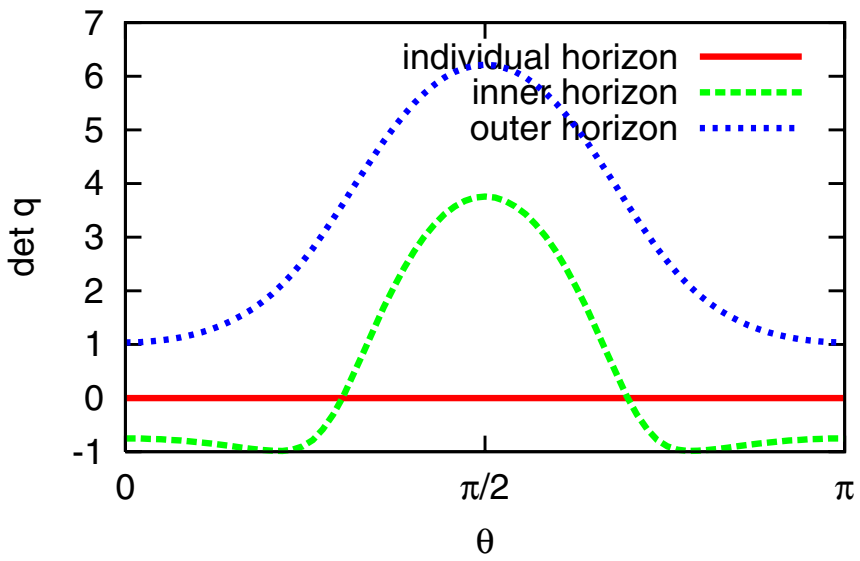

(a) $t=0.6$
Horizon metric determinant at $\mathrm{t}=1$

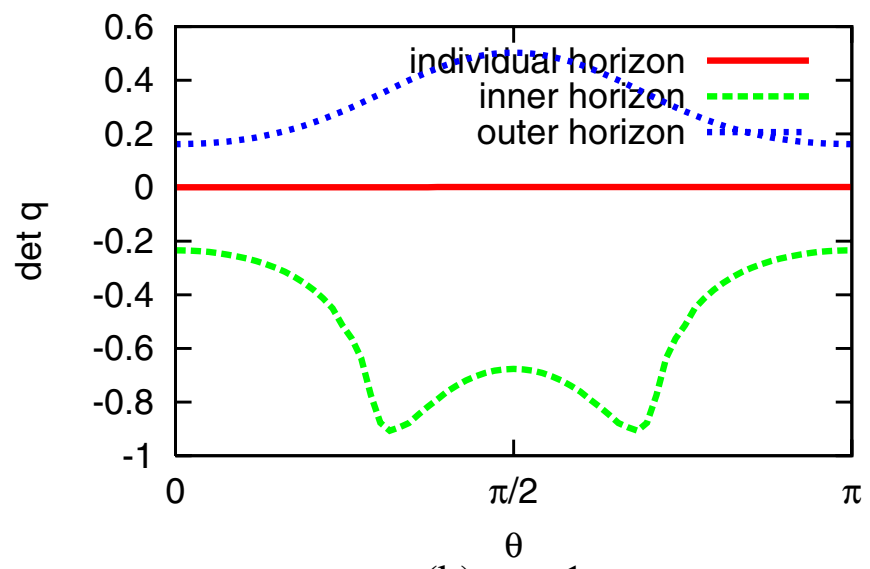

(b) $t=1$

FIG. 4 (color online). Determinant of the horizon world tube's three-metric vs latitude $\theta$ at $t=0.6$ and $t=1$. The individual MTTs are null, i.e., $\operatorname{det} \tilde{q}=0$ (up to numerical errors). The common outer MTT is spacelike (i.e., $\operatorname{det} \tilde{q}>0$ ) and it tends to null at late times. The inner common MTT is partially timelike at $t=0.6$; later it becomes completely timelike.

used in the apparent horizon finder is inaccurate near the neck of the inner horizon [48]. Figure 5 shows the time evolution of the masses $M=\sqrt{A_{S} / 16 \pi}$ of the individual and the common horizons (in this case, the angular momentum vanishes identically). If $M^{\infty}$ is the asymptotic value of the mass of the outermost horizon at late times, then $M_{\mathrm{ADM}}-M^{\infty}$ is, in principle, a reliable way of estimating the amount of energy radiated away to infinity in the form of gravitational waves. This difference could be used as a consistency check on other estimates using the extracted waveforms at large distances from the black holes. However, our emphasis in this paper is on the dynamics of the merger and not on long duration stable evolutions. Our simulations do not last long enough to estimate $M^{\infty}$ reliably.

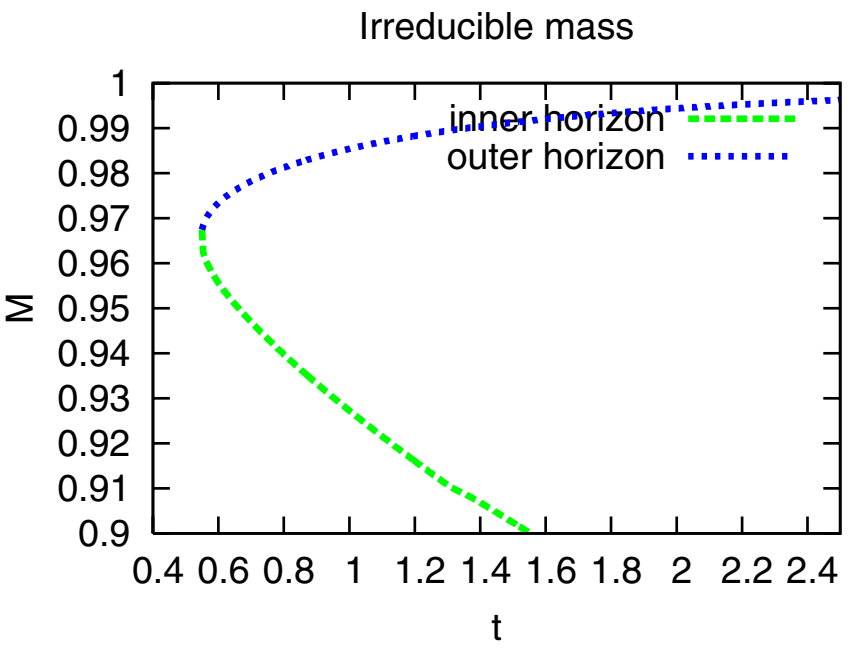

FIG. 5 (color online). Irreducible mass vs time for the individual and the common MTTs. The outer common MTT grows and accretes mass, while the inner MTT shrinks and loses mass.
Another feature of the horizons, shown in Fig. 5, is that while the common outer MTT increases in area as expected, the area of the common inner MTT decreases monotonically. This is explained as follows. Initially, when the common MOTS is just formed, by continuity with the outer MTT, the inner MTT is spacelike for a very short duration (much before $t=0.6$ ) and it is thus a DH for this duration. However, this DH is being traversed in the inwards direction (i.e., along $-\hat{r}^{a}$ ) so that its area appears to decrease. Shortly after its formation, the inner MTT becomes partly timelike and later fully timelike. Recall that for a TLM, the area decreases if $\Theta_{(n)}<0$. Thus, both the spacelike and timelike portions of the inner MTT contribute to its monotonic area decrease. This behavior of the outer MTT is roughly similar to what was found in [47] for spherically symmetric horizons; however due to spherical symmetry, the horizons in [47] did not have any cross sections of mixed signature.

Figure 6 demonstrates how the common outer apparent horizon grows. The energy flux vanishes at the poles, and the shear (but not the total flux) is maximum at the equator. The horizon is spacelike all the time, but it becomes increasingly isolated at late times as it approaches equilibrium. Thus the rate of area increase becomes smaller and the fluxes also becomes correspondingly smaller.

Let us now consider the higher mass multipoles $M_{n}$ (all the $J_{n} \mathrm{~s}$ vanish identically). Here, since all quantities are symmetric with respect to a reflection about the equatorial plane, $M_{n}=0$ for odd $n$. Figure 7 plots the mass quadrupole moment $M_{2}$ and also $M_{4}$ and $M_{6}$ of the outer and inner common MTTs as a function of time. We expect that the black hole should eventually settle down to a Schwarzschild solution by radiating away all of its higher multipole moments. Clearly, for the outer MTT, $M_{2}, M_{4}$ and $M_{6}$ all become smaller with time, approaching zero. 

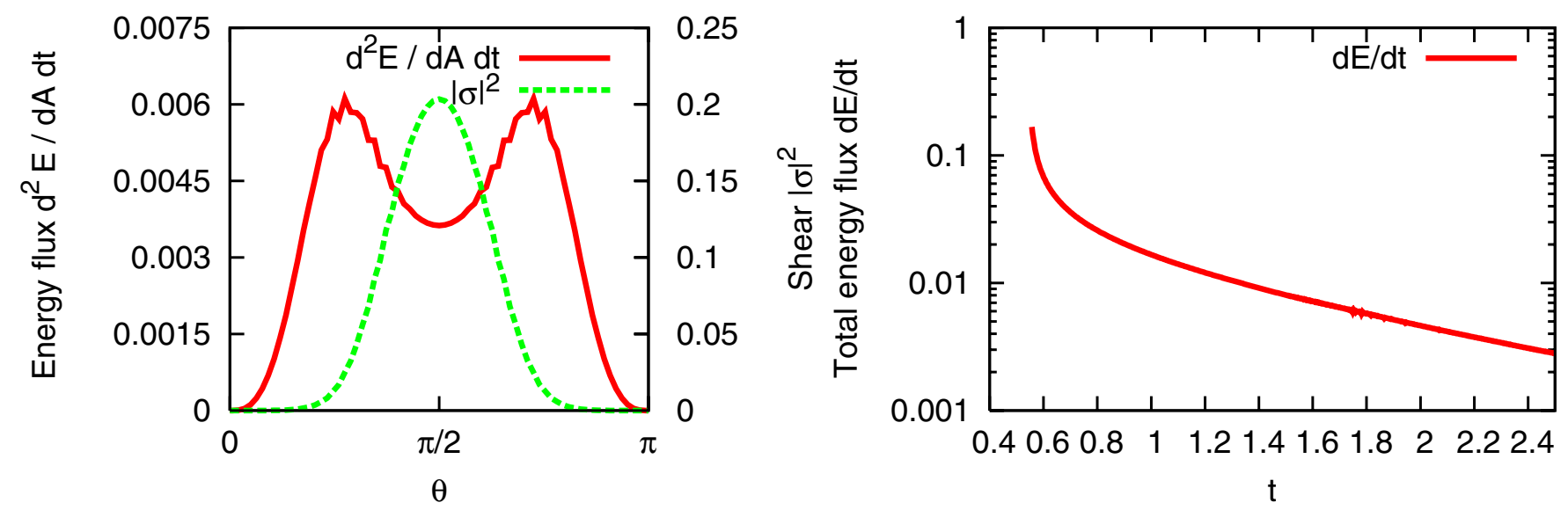

FIG. 6 (color online). Energy flux and shear $\left|\sigma_{(\bar{\ell})}\right|^{2}$ through the outer common horizon vs latitude $\theta$ at $t=0.6$, and the total energy flux vs time. The shear vanishes at the poles and the black hole settles down exponentially.

Outer horizon

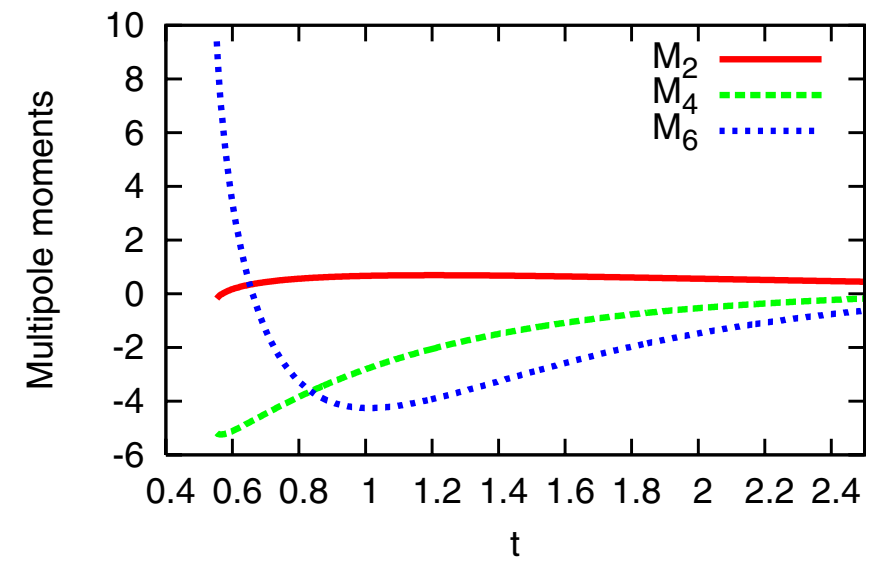

Inner horizon

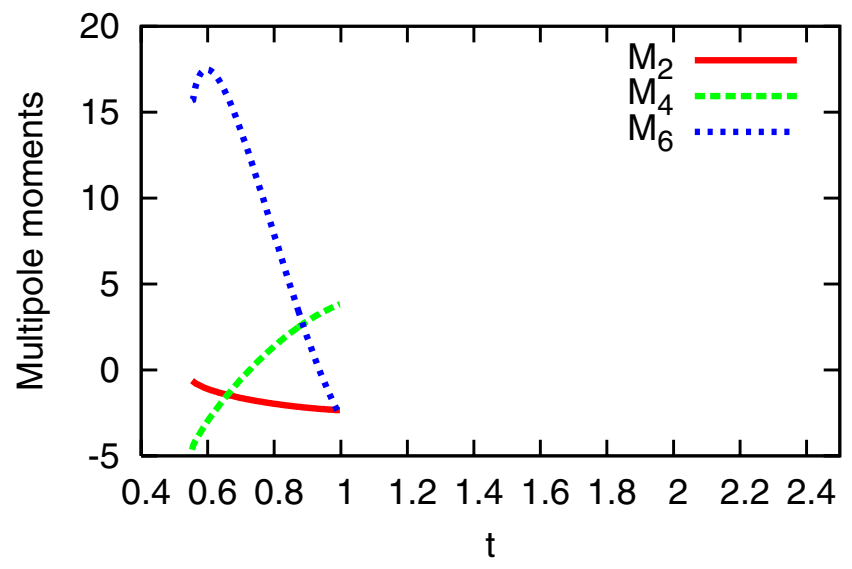

FIG. 7 (color online). Some mass multipole moments vs time for the inner and outer MTTs for the head-on collision. The multipole moments for the outer horizon all approach their Schwarzschild values (i.e., 0) but the inner horizon does not seem to do so.

However, the run did not last long enough for us to obtain the asymptotic falloff rate. It is interesting to note that, as far as we can tell, the multipole moments for the inner MTT do not vanish asymptotically. This tells us that the spacetime near the inner MTT is not close to Schwarzschild even at late times. At even later times, all the inner horizons presumably cease to exist (see next paragraph) and the spacetime approaches Schwarzschild everywhere.

We conclude this section with some remarks on the eventual fate of the inner MTT. First of all, as expected, the outer MTT eventually settles down and approaches future timelike infinity. The inner MTT shrinks and approaches the two individual horizons which are essentially stationary. It is interesting to speculate on how, if at all, the inner MTT will merge with the two individual MTTs. Does the inner MTT "pinch off" into two individual horizons?
If the inner MTT is indeed the one predicted by [52], then it has a priori curvature bounds. If these curvature bounds are maintained in the limit, then the inner horizon cannot pinch off. It is more likely that the two individual MTTs merge first with each other and then later, perhaps also with the inner MTT. It would be interesting to investigate this question further. If the inner MTT does indeed merge smoothly with the two individual MTTs, then the set of all MTTs in this case would form one single smooth 3manifold. Furthermore, the area of the cross section of this manifold would be monotonic in the outward directiontraversing this manifold in the outward direction means going forward in time on the individual and outer MTTs, and backward in time on the inner MTT.

We are not able to settle these issues numerically in a conclusive manner because the inner MTT becomes so distorted at late times that the $\mathrm{AH}$ tracker is no longer 
able to track it. This is because the AH tracker can only locate star-shaped surfaces and, as is clear from Fig. 3, the inner MTT will not necessarily be star-shaped at later times. Furthermore, our gauge choice in which we allow the outer MTT to grow in coordinate space, makes the inner MTT shrink and therefore harder to resolve at later times.

\section{B. Nonaxisymmetric black hole collision}

The head-on collision described above does not incorporate any effects of angular momentum. In this section, we remove the restriction of axisymmetry by taking initial configurations in which the black holes are orbiting around each other. We use the so called "puncture" data introduced by Brandt and Brügmann [66], which is a generalization of the Brill-Lindquist construction. The data is still taken to be conformally flat, but now no longer assumed to be time symmetric.

We performed a numerical evolution of puncture initial data corresponding to the innermost stable circular orbit as predicted in [67]. This model was also studied as "QC-0" with the Lazarus perturbative matching technique $[68,69]$ and later in $[2-5,70]$. In our setup, the punctures were located at $x= \pm 1.168642873$, and their mass parameters were $m=0.453$, and their momenta were $p_{y}=$ \pm 0.3331917498 . The domain had an explicit rotating quadrant symmetry and extended up to $x, y, z=10$. Near the outer boundary the spatial resolution was $h=0.4$, and near the punctures we used mesh refinement to increase the resolution successively up to $h=0.025$, so that the individual horizon diameters contained initially 16 grid points. We used fourth order accurate spatial differencing operators, and a third order Runge-Kutta time integrator.

We excised [58] coordinate spheres with a radius of $r_{e}=$ 0.075 about the punctures from the domain, corresponding to a diameter of 6 grid points. We used again the AEI BSSN formulation [58,59] for time evolution, a $1+\log$ slicing condition [60] starting from a lapse that is one at infinity and zero at the punctures, and a $\Gamma$ driver shift condition, starting from a rigid corotation with an angular velocity of $\omega=0.06$. We also used a drift correcting shift term similar to $[71,72]$ to keep the individual horizons centered about their initial locations.

As previously, we used the CACTUS framework $[61,62]$, the Carpet mesh refinement driver [63,64], and the CACTUSEINSTEIN infrastructure. We solved the initial data equation with M. Ansorg's TWOPUNCTURE solver [73], and we located the apparent horizon surfaces with J. Thornburg's AHFINDERDIRECT [48].

This setup contains two initially separated horizons that rotate around each other for a fraction of an orbit before a common horizon forms [68,70]. Its ADM mass is $M_{\mathrm{ADM}}=$ 1.00788 , the initial proper horizon separation is $L \approx$ $4.99 M_{\mathrm{ADM}}$, and the horizons have initially the angular momentum $J \approx 0.78 M_{\mathrm{ADM}}^{2}$ and angular velocity $\Omega \approx$
Horizon shapes at $\mathrm{t}=18$

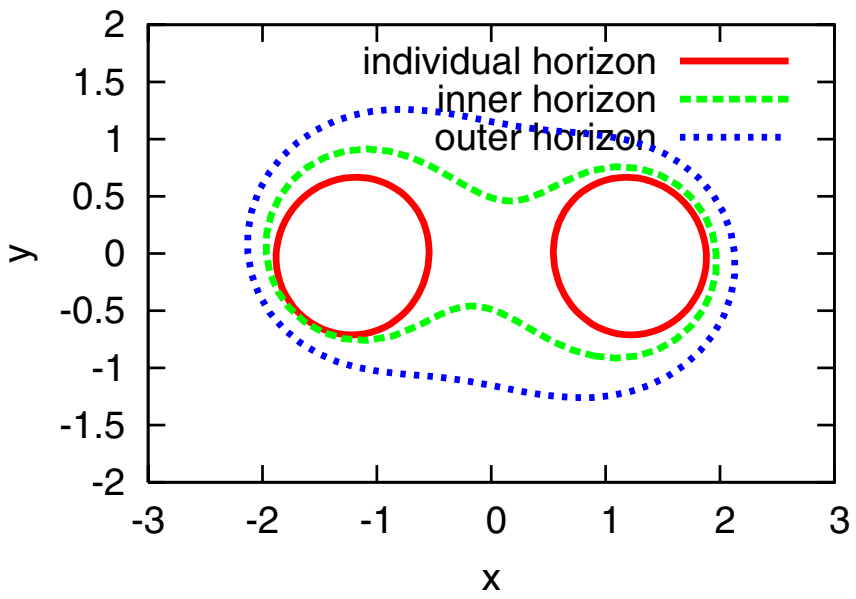

FIG. 8 (color online). Coordinate shapes of the MOTSs at $t=$ 18 for the nonaxisymmetric black hole collision. Note that the individual horizons are locked in place through the corotating coordinate system and through an adaptive shift condition.

$0.17 / M_{\mathrm{ADM}}$. The common apparent horizon forms at about $t=17.5$, which we verified through pretracking [74].

Figure 8 shows the shape of the various MOTSs at a time $t=18$, a short while after the common horizon has formed. The qualitative behavior of the various MTTs is exactly the same as in Sec. IVA2. Figure 9 compares the determinants of the MTT three-metrics at this time. Figure 10 shows the irreducible mass of the outer and inner MTTs as a function of time. Again, the behavior is qualitatively the same as we saw in the head-on collision.

Figure 11 shows the flux of gravitational wave energy falling into the outer horizon at $t=18.4$ and also the shear

\section{Horizon metric determinant at $\mathrm{t}=18$}

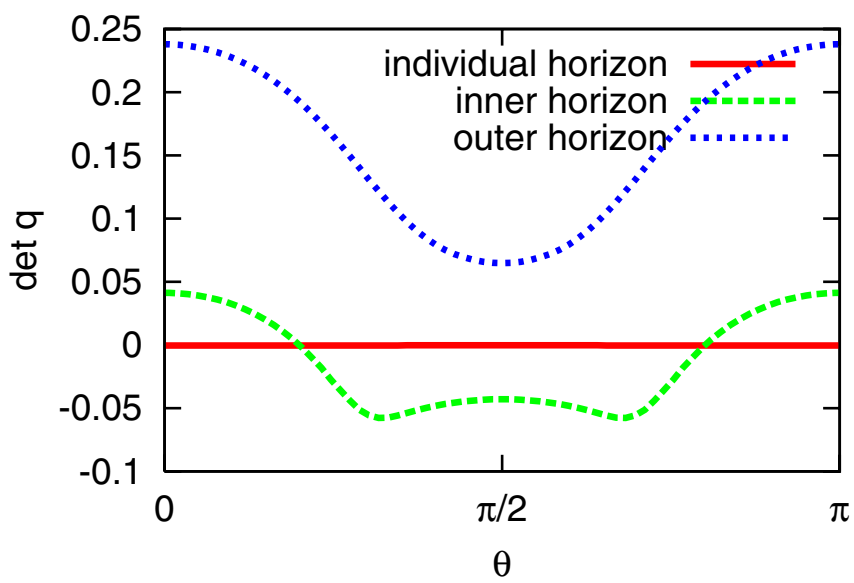

FIG. 9 (color online). Determinant of the MTT three-metric at $t=18$. As in the head-on case, the outer MTT is purely spacelike while the inner MTT is partly spacelike and partly timelike. At later times, it becomes purely timelike. The individual MTTs are null at this time. 
Irreducible mass

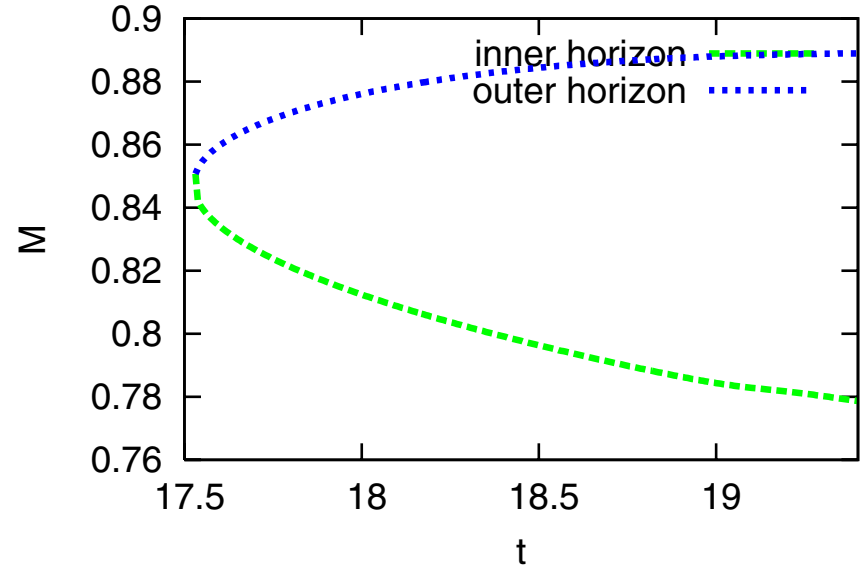

FIG. 10 (color online). A plot of the irreducible mass $M_{\text {irr }}=$ $\sqrt{A / 16 \pi}$ as a function of time for the outer an inner MTTs in the nonaxisymmetric black hole collision. As expected, the outer MTT has increasing area while the inner MTT shrinks.
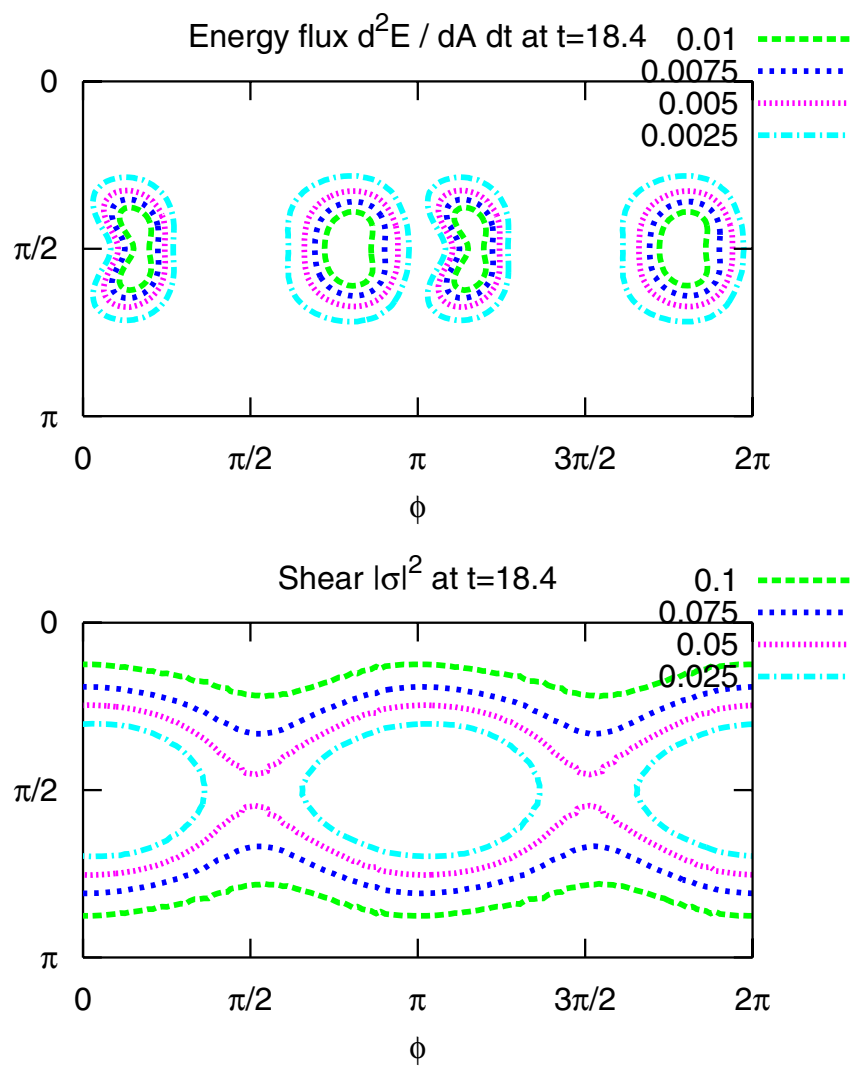

FIG. 11 (color online). Energy flux through the common horizon and shear $\left|\sigma_{(\bar{\ell})}\right|^{2}$ on the horizon at $t=18.4$ in $(\theta, \phi)$-coordinates. The spacetime is not axisymmetric, and since it contained two inspiralling black holes, there is no mirror symmetry across the $x-z$ or $y-z$ planes either. The energy flux shows this asymmetry clearly. $\left|\sigma_{(\bar{\ell})}\right|^{2}$ at the same time, for the outer and individual horizons. The 2-d contour plots of the shear $\left|\sigma_{(\bar{\ell})}\right|^{2}$ and the total flux on the horizon shows in detail how gravitational radiation is falling into the horizon. Unlike in the head-on case (Fig. 6), the shear and the flux are now no longer axisymmetric. Therefore, the flux is no longer constant along the $\phi$ direction but its maxima still lie on the equator. The shear on the other hand, now has its maximum on the poles and its minima lie on the equator. It would be interesting to further investigate the behavior of $\left|\sigma_{(\bar{\ell})}\right|^{2}$ and the energy flux as a function of time and for different physical situations to gain a better understanding of how a black hole grows.

Let us now turn to the rotational vector $\varphi^{a}$ on the outer horizon and the quantities such as angular momentum, mass, and multipole moments associated with it. The simulation presented here was run only up to $t \approx 19.4$, and the final black hole has not settled down sufficiently, and has not attained axisymmetry at this point. Figure 12 shows the Lie derivative of the 2-metric $\mathcal{L}_{\varphi} \tilde{q}_{a b}$ on the horizon at $t=18$, where $\varphi^{a}$ is the Killing vector candidate found by the algorithm presented in [35]. It is clear that $\mathcal{L}_{\varphi} \tilde{q}_{a b}$ is very far from 0 at this time. This means that the angular momentum, mass, and multipole moments associated with this $\varphi^{a}$ are not meaningful at this point. This is to be expected, since the final black hole should attain axisymmetry only on a time scale set by the quasinormal mode ringdown, which has a period of $15.9 M_{\mathrm{ADM}}$ in this case. It is interesting to see that our Killing vector field

\section{Lie derivative of the two-metric at $t=18$}

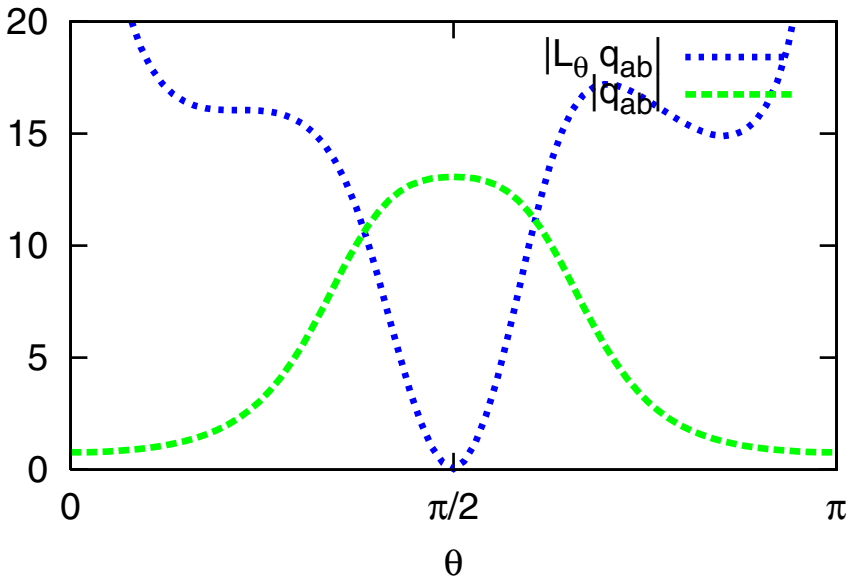

FIG. 12 (color online). Lie derivative of the two-metric $\mathcal{L}_{\varphi} \tilde{q}_{a b}$ at $t=18$ on the $\phi=0$ line. The two-metric $\tilde{q}_{a b}$ is also shown for comparison. The quantity shown in the plots are actually the norms $\sqrt{\sum_{a b}\left(\mathcal{L}_{\varphi} \tilde{q}_{a b}\right)^{2}}$ and $\sqrt{\sum_{a b}\left(\tilde{q}_{a b}\right)^{2}}$ in the coordinate system $(\theta, \phi)$ on the horizon. The vector field $\varphi$ is Killing on the equator (see main text), but not everywhere. This shows that the horizon is not (yet) axisymmetric. We expect it to become axisymmetric at later times. Note that we have only shown the plots along the $\phi=0$ curve and we do not have axisymmetry here. 
candidate is indeed Killing on the equator. This is by construction, since we choose the Killing vector field candidate by an integral along the equator; see [35]. However, the vector field $\varphi^{a}$ is far from Killing away from the equator.

A word of caution is due here regarding the Killing vector finding algorithm of [35]. First of all, the algorithm only produces a candidate for a Killing vector, and an independent check is required to see whether $\mathcal{L}_{\varphi} \tilde{q}_{a b}$ is sufficiently small or not. Furthermore, as mentioned previously, this method reduces the problem of finding a Killing vector on a sphere to diagonalizing a $3 \times 3$ matrix followed by integrating a 1-dimensional ODE. In particular, the method requires that one of the eigenvalues of this matrix is sufficiently close to unity. While this is fine when the horizon is exactly axisymmetric, the subtlety arises when the horizon is only approximately axisymmetric. It is not clear how close the eigenvalue must be to unity for the horizon to be regarded as approximately axisymmetric. Work is in progress to understand this better and to also investigate an alternate method of finding an appropriate $\varphi^{a}$ as discussed in Sec. III B, which is guaranteed to produce a divergence free vector.

\section{Axisymmetric gravitational collapse}

\section{The initial configuration}

Up to now, all of our examples have involved only vacuum spacetimes. In this section, we present an example of the gravitational collapse of a neutron star to form a black hole in an axisymmetric spacetime. These simulations were performed using the WHISKY code which deals with the matter terms of the Einstein equations in the framework of the CACTUS toolkit. Thus, the WHISKY code solves the conservation equations for the stress energy tensor $T_{a b}$ and for the matter current density $J^{a}$ :

$$
\nabla^{a} T_{a b}=0, \quad \nabla_{a} J^{a}=0 .
$$

For details about the WHISKY code and the implementation of the above equations, we refer the reader to [75] and references therein. Here we shall restrict ourselves to describing the initial stellar configuration which is one of the configurations studied in [75].

The neutron star is modeled as a uniformly rotating ball of perfect fluid. The equation of state is taken to be a $K=$ $100, \Gamma=2$ polytrope so that the pressure $p$ and rest-mass density $\rho$ are related according to $p=K \rho^{\Gamma}$. The equilibrium configuration is determined by the mass $M_{\mathrm{NS}}$, central density $\rho_{c}$, and the angular momentum $J_{\mathrm{NS}}$; when necessary, the subscript NS is used in order to avoid any confusion with previously defined symbols. The model we take is the one denoted as "D4" in [75] which has $M_{\mathrm{NS}}=$ $1.86 M_{\odot}, \quad \rho_{c}=1.934 \times 10^{15} \mathrm{~g} \mathrm{~cm}^{-3}, \quad$ and $J_{\mathrm{NS}}=$ $0.543 M_{\mathrm{NS}}^{2}$. This leads to a ratio of polar to equatorial coordinate radii of 0.65 , a circumferential equatorial radius of $14.22 \mathrm{~km}$, and a rotational frequency of $1295.34 \mathrm{~Hz}$.
This equilibrium configuration turns out to be dynamically unstable. In practice, the instability is induced by uniformly reducing the pressure slightly throughout the star.

\section{Numerical results}

We simulated the above system on a grid with an explicit rotating octant symmetry. The outer boundary was at $x, y, z=150$, and the grid spacing near the outer boundary was $h=3$. We used mesh refinement to increase our spatial resolution in the center of the domain to $h=$ 0.375 at the initial time, and progressively introduced more mesh refinement levels to increase the central resolution up to $h=0.046875$ as the neutron star collapsed, based on the maximum density in the star [76,77]. We also apply third order Kreiss-Oliger dissipation [78] to the spacetime (but not the hydrodynamics) variables.

We find an apparent horizon starting at about $t=130$; this time is mainly dependent on the details of how the collapse is induced and has no intrinsic meaning. The horizon is born with an irreducible mass of about $M_{\text {irr }}=$ 1.51 and an angular momentum of $J=0.89(a=0.38)$, giving it a total mass of $M_{H}=1.54$. Some time after $t=$ 185 , a singularity forms in the spacetime, and the simulation aborts because we do not use excision inside the apparent horizon. As before, a pair of MOTSs is formed, an outer and an inner one. The outer MTT is spacelike, has increasing area, and tends to null at late times. In this case, the inner MTT remains spacelike. However, its area decreases because we are traversing it in the inward direction; in other words, the time evolution vector $t^{a}$ is such that at the inner MTT, $t \cdot \hat{r}<0$ so that the area decreases along $t^{a}$. Our gauge conditions are such that the outer horizon grows in coordinate space while the inner horizon shrinks. After about $t=140$, the inner horizon is so small that we do not have enough resolution to track it beyond that time. See Fig. 13. The areal radius of the outer MTT increases but not as rapidly as the coordinate radius; it levels off at later times. The area radius of the inner horizon decreases initially and shows an increase at later times, but this is probably just a numerical artefact due to poor resolution at later times.

Figure 14 shows the determinant of the metric on the MTTs. The outer MTT is initially spacelike, which is consistent with its growing, and exponentially approaches null at late times. After about $t=160$, the simulation cannot distinguish the horizon world tube signature from null any more. As an example we also show the determinant as a function of the latitude $\theta$ at $t \approx 138$, and the average value of the determinant over the horizon as a function of time. The inner MTT is also spacelike and becomes more and more null at least as long as we are able to track it reliably.

Figure 15 shows the outer horizon has grown at $t=155$ to an irreducible mass of $M_{\mathrm{irr}}=1.80$ and an angular momentum of $J=1.93(a=0.55)$, giving it a total mass 


\section{Horizon radii}

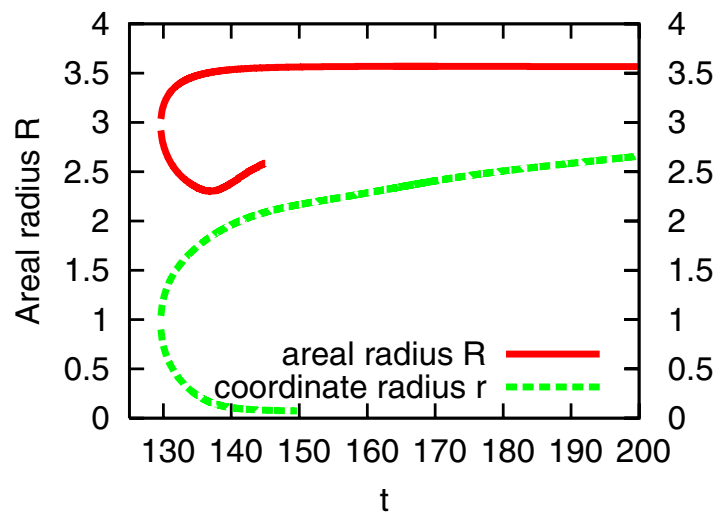

FIG. 13 (color online). The average coordinate radius and the area radius as a function of time for the outer and inner MTTs for the neutron star collapse. The inner horizon is not to be trusted after $t \approx 140$ due to lack of resolution, since its coordinate radius has become very small by that time.

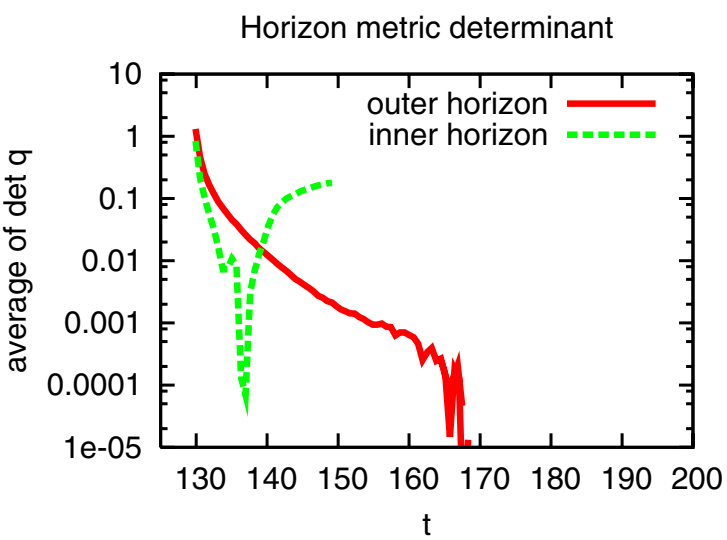

of $M_{H}=1.87$. For comparison, the corresponding ADM quantities are $M_{\mathrm{ADM}}=1.86$ and $J_{\mathrm{ADM}}=1.88(a=0.54)$. Because the spacetime is axially symmetric, gravitational waves cannot carry away angular momentum. That means that the spin $a=J / M^{2}$ is approximately correct at late times. Unlike in the nonaxisymmetric black hole collision discussed earlier, the present case is explicitly axisymmetric and there are no problems with locating the rotational symmetry vector.

Figure 16 shows the mass quadrupole moment $M_{2}$ and the angular momentum octopole moment of the outer and inner MTTs as a function of time. Given that we know the asymptotic values of the area and angular momentum of these MTTs (the ADM values), we can also calculate the expected values of $M_{2}$ and $J_{3}$ at late times. The plots clearly show that the values of $M_{2}$ and $J_{3}$ approach the Kerr values at later times (though this matching is not exact, presumably due to numerical errors). Also note that $M_{2}$ is noisy. We have observed such noise only in simulations that include matter, and we find that this noise

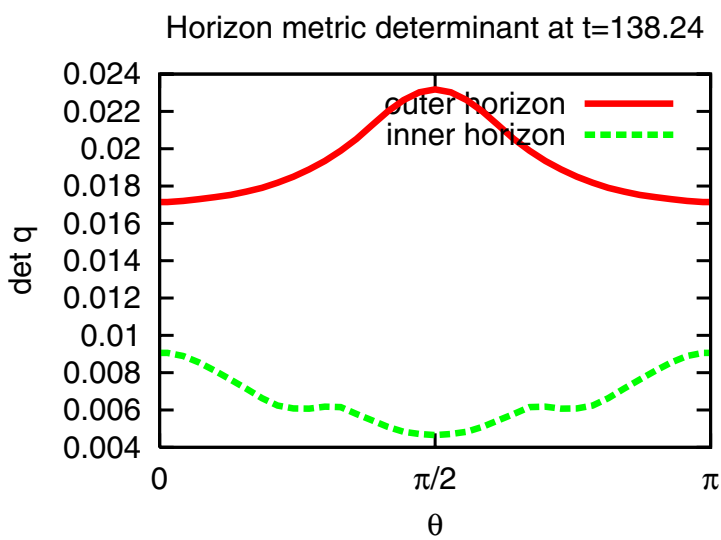

FIG. 14 (color online). Average of the determinant of the horizon world tube's three-metric vs time, and vs latitude $\theta$ at $t=138.24$ for the inner and outer horizons for the neutron star collapse.
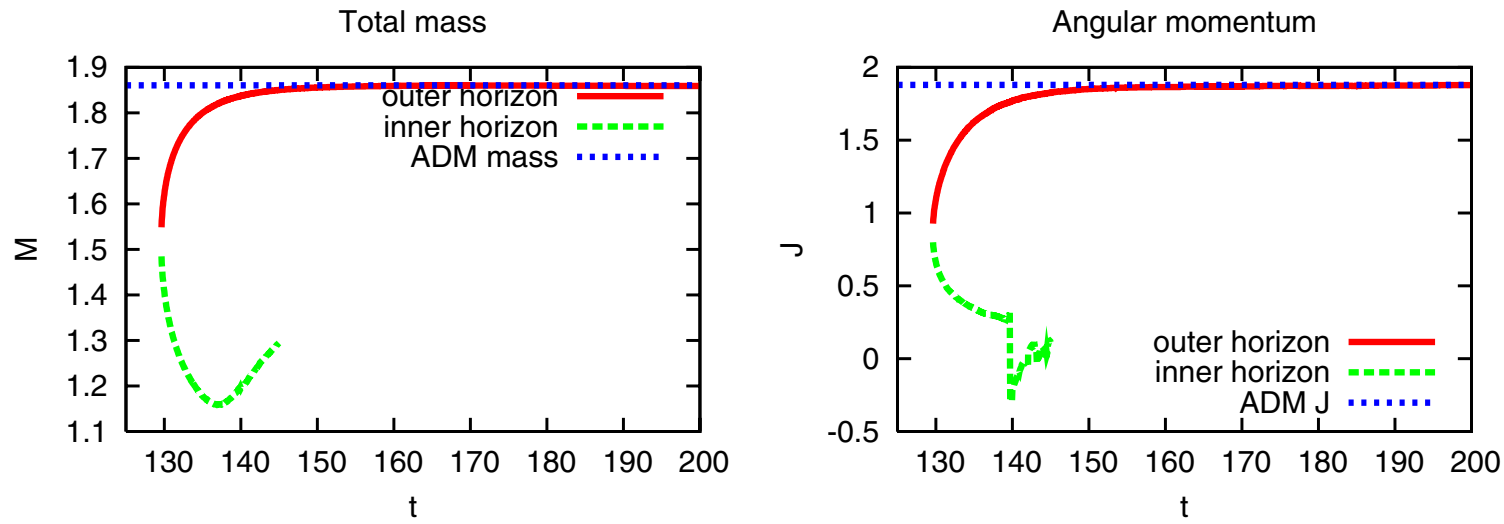

FIG. 15 (color online). The total mass $M_{H}$, and angular momentum $J$ as a function of time for the outer and inner MTTs for the neutron star collapse. 
Mass quadrupole moment

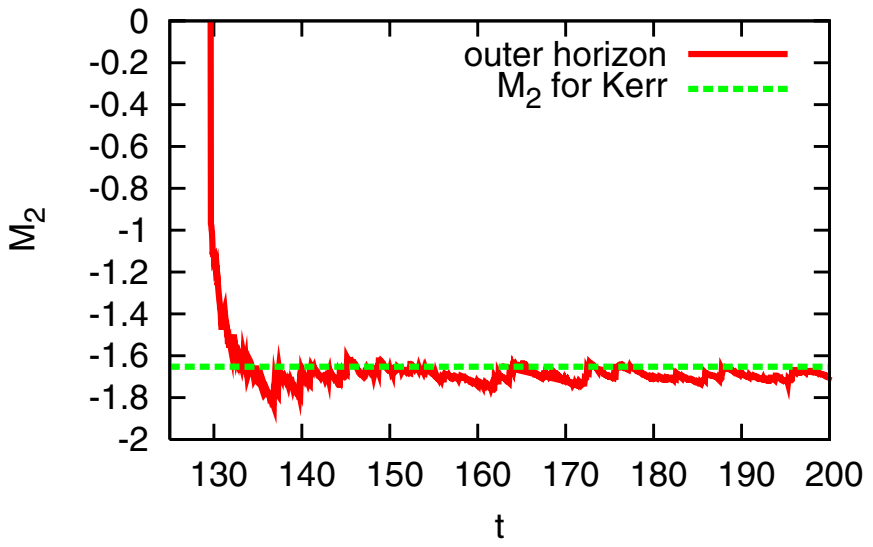

Angular momentum octupole moment

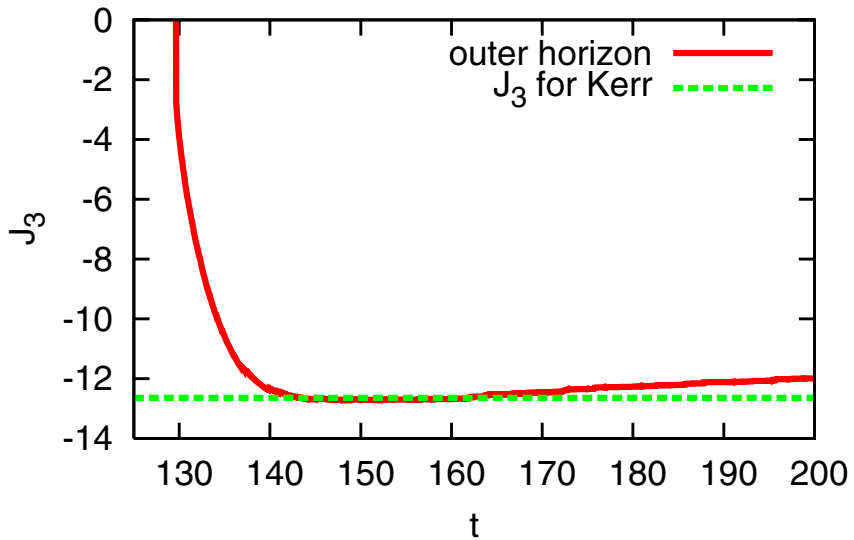

FIG. 16 (color online). Horizon mass quadrupole moment $M_{2}$ and angular momentum octopole moment $J_{3}$ vs time for the neutron star collapse. For comparison, the values for a Kerr black hole with the same ADM mass and angular momentum as the initial data are also shown.

is much improved by using artificial dissipation on the spacetime variables (which we do). The angular momentum multipoles seem unaffected.

\section{DISCUSSION}

In this article, we have applied the dynamical horizon formalism to numerical simulations of black hole spacetimes. The main theme in this formalism is to take trapped surfaces seriously as a way of describing black hole physics. Marginally trapped surfaces behave more regularly that one might have expected previously, and they are useful for extracting interesting physical information about the horizon. We have shown how the mass, angular momentum, multipole moments, and the flux of energy due to in-falling gravitational radiation and matter can be calculated in a coordinate independent way (given a particular time slicing of our spacetime). We have implemented these ideas numerically and shown three concrete examples. In these examples, we see how the black hole is formed, how it grows, and how it settles down to an isolated Kerr black hole. We have also seen that the dynamical horizon formalism is valuable for exploring the geometry of the trapped region. It allows us to classify various types of trapped surfaces which might appear during the course of a gravitational collapse or a black hole coalescence. Finally, these ideas can also be viewed as a set of diagnostic tools which allow us to keep track of what is going on during the course of a numerical simulation, and whether numerical results make sense and satisfy some basic, but nontrivial properties in the strong field region.

Some suggestions for future work:

(i) As mentioned in the text, the calculation of the axial vector $\varphi^{a}$ for nonaxisymmetric cases is not yet satisfactory. We have used the method sug- gested in [35] which works well enough at early and late times, when the horizon is approximately axisymmetric. However, in general, the result is not guaranteed to be divergence free and thus the angular momentum not guaranteed to be gauge invariant. We have not yet implemented the generalization described in Sec. III B satisfactorily; this is work in progress.

(ii) The accuracy of the numerical examples that we have shown decreases with time, and this is a common feature of most present day black hole numerical simulations. Thus, we have not been conclusively able to prove that the black hole settles down to Kerr (though there are strong indications that this does happen). We have not been able to extract the rate at which equilibrium is reached, thereby extending Price's law (see [79] and e.g. [80]) to more general situations, but this is, in principle possible and requires more stable and accurate simulations. Similarly, we have not been able to accurately calculate the asymptotic value of the black hole mass $M^{\infty}$. The difference $M_{\mathrm{ADM}}{ }^{-}$ $M^{\infty}$ is, in principle, a reliable estimate of the amount of energy radiated to infinity. While the ADM mass is hard to calculate reliably during the simulation because of the finite grid and low resolution in the asymptotic region, it can usually be calculated accurately from the initial data themselves. Calculating $M^{\infty}$ and understanding this estimate of the radiated energy requires more accurate and stable runs, applied to diverse and realistic initial data. The results of [41] could also be used to study the approach to equilibrium.

(iii) It would be useful numerically to have a gauge condition which ensures that the horizon stays at the same coordinate location at all times. While 
such conditions are not difficult to find in the isolated case, dynamical situations are harder. Given the location of an outer MOTS at a particular instant of time, the results and methods of [34] can be used to predict the location of the MOTS at the next instant by solving an elliptic equation on the MOTS. This could be used to construct appropriate gauge conditions and evolution schemes which take the horizon geometry into account $[49,81]$.

(iv) What happens to the inner horizon of Figs. 2, 3, and 8? As described in Sec. IVA 2, the eventual fate of these inner MTTs and the two individual horizons is not yet known, and would be interesting to investigate further. This requires simulations with higher resolution near the inner horizons, different gauge conditions, and perhaps also $\mathrm{AH}$ trackers capable of handling non-star-shaped surfaces, and perhaps also higher genus surfaces.

(v) Can the methods of [34] be extended for MOTSs which are not strictly-stably-outermost? In this regard, it would be interesting to study the stability operator $L_{\Sigma}$ introduced in [34]. For a strictly-stably-outermost MOTS, the principle eigenvalue of $L_{\Sigma}$ turns out to be strictly positive and this is an important ingredient in the existence results. A numerical computation of the eigenvalues of this operator, especially during the transition between inner and outer MTTs and for the inner nonspacelike MTTs might lead to further insights.

\section{ACKNOWLEDGMENTS}

We are grateful to Lars Andersson and Abhay Ashtekar for many valuable suggestions and fruitful discussions. We also thank Ivan Booth, Sergio Dain, Steve Fairhurst, Greg Galloway, Ian Hawke, Sean Hayward, Jan Metzger, Denis Pollney, Reinhard Prix, Jonathan Thornburg, and Robert Wald for useful discussions. As always, our numerical calculations would have been impossible without the large number of people who made their work available to the public: we used the CACTUS framework [61,62] and the CACTUSEINSTEIN infrastructure [82] with a number of locally developed thorns, such as the initial data solver TWOPUNCTURES by M. Ansorg, the mesh refinement criteria set up via WHISKYCARPETREGRID by C. D. Ott and I. Hawke, and the horizon finder AHFINDERDIRECT by $\mathrm{J}$. Thornburg. We also used the general relativistic hydrodynamics code WHISKY [83] developed by the authors of [75], and the initial data generator RNSID by N. Stergioulas, which were both developed during the EU training network "Sources of Gravitational Waves." The code uses routines of the LAPACK $[84,85]$ and BLAS [86] libraries from the Netlib Repository [87], the Numerical Recipes [88], and the UMFPACK [89] library. The numerical simulations were performed on the Peyote Beowulf Cluster at the AEI. ES was partly funded by the DFG's special research center SFB TR/7 "Gravitational Wave Astronomy." This work was supported by the Albert-Einstein-Institut and the Center for Computation and Technology at LSU.
[1] F. Pretorius, Phys. Rev. Lett. 95, 121101 (2005).

[2] M. Campanelli, C. O. Lousto, P. Marronetti, and Y. Zlochower, Phys. Rev. Lett. 96, 111101 (2006).

[3] J. G. Baker, J. Centrella, D.-I. Choi, M. Koppitz, and J. van Meter, Phys. Rev. Lett. 96, 111102 (2006).

[4] F. Herrmann, D. Shoemaker, and P. Laguna, gr-qc/ 0601026.

[5] M. Campanelli, C. O. Lousto, and Y. Zlochower, Phys. Rev. D 73, 061501(R) (2006).

[6] J. G. Baker, J. Centrella, D.-I. Choi, M. Koppitz, and J. van Meter, Phys. Rev. D 73, 104002 (2006).

[7] P. Diener, F. Herrmann, D. Pollney, E. Schnetter, E. Seidel, R. Takahashi, J. Thornburg, and J. Ventrella, Phys. Rev. Lett. 96, 121101 (2006).

[8] M. Campanelli, C. O. Lousto, and Y. Zlochower, gr-qc/ 0604012.

[9] A. Ashtekar and B. Krishnan, Phys. Rev. Lett. 89, 261101 (2002).

[10] A. Ashtekar and B. Krishnan, Phys. Rev. D 68, 104030 (2003).

[11] A. Ashtekar, C. Beetle, and S. Fairhurst, Classical Quantum Gravity 16, L1 (1999).

[12] A. Ashtekar, C. Beetle, and S. Fairhurst, Classical
Quantum Gravity 17, 253 (2000).

[13] A. Ashtekar, C. Beetle, and J. Lewandowski, Phys. Rev. D 64, 044016 (2001).

[14] A. Ashtekar, S. Fairhurst, and B. Krishnan, Phys. Rev. D 62, 104025 (2000).

[15] A. Ashtekar, C. Beetle, O. Dreyer, S. Fairhurst, B. Krishnan, J. Lewandowski, and J. Wisniewski, Phys. Rev. Lett. 85, 3564 (2000).

[16] S. A. Hayward, Phys. Rev. D 49, 6467 (1994).

[17] S. Hayward, Classical Quantum Gravity 11, 3025 (1994).

[18] S. A. Hayward, Phys. Rev. D 70, 104027 (2004).

[19] A. Ashtekar and B. Krishnan, Living Rev. Relativity 7, 10 (2004).

[20] I. Booth, Can. J. Phys. 83, 1073 (2005).

[21] E. Gourgoulhon and J. L. Jaramillo, Phys. Rep. 423, 159 (2006).

[22] P. Diener, Classical Quantum Gravity 20, 4901 (2003).

[23] S. A. Hughes, C. R. Keeton, II, P. Walker, K. T. Walsh, S. L. Shapiro, and S. A. Teukolsky, Phys. Rev. D 49, 4004 (1994).

[24] P. Anninos, D. Bernstein, S. R. Brandt, D. Hobill, E. Seidel, and L. Smarr, Phys. Rev. D 50, 3801 (1994).

[25] P. Anninos, D. Bernstein, S. Brandt, J. Libson, J. Massó, E. 
Seidel, L. Smarr, W.-M. Suen, and P. Walker, Phys. Rev. Lett. 74, 630 (1995).

[26] S. R. Brandt and E. Seidel, Phys. Rev. D 52, 870 (1995).

[27] J. Libson, J. Massó, E. Seidel, W.-M. Suen, and P. Walker, Phys. Rev. D 53, 4335 (1996).

[28] J. Massó, E. Seidel, W.-M. Suen, and P. Walker, Phys. Rev. D 59, 064015 (1999).

[29] R.M. Wald, General Relativity (The University of Chicago Press, Chicago, 1984), ISBN 0-226-87032-4 (hardcover), 0-226-87033-2 (paperback).

[30] R. Penrose, Phys. Rev. Lett. 14, 57 (1965).

[31] R. Penrose and S. W. Hawking, Proc. R. Soc. A 314, 529 (1970).

[32] S. W. Hawking and G.F. R. Ellis, The Large Scale Structure of Spacetime (Cambridge University Press, Cambridge, England, 1973), ISBN .

[33] M. Kriele and S. A. Hayward, J. Math. Phys. (N.Y.) 38, 1593 (1997).

[34] L. Andersson, M. Mars, and W. Simon, Phys. Rev. Lett. 95, 111102 (2005).

[35] O. Dreyer, B. Krishnan, D. Shoemaker, and E. Schnetter, Phys. Rev. D 67, 024018 (2003).

[36] S. Dain, J. L. Jaramillo, and B. Krishnan, Phys. Rev. D 71, 064003 (2005).

[37] J. L. Jaramillo, E. Gourgoulhon, and G. A. Mena Marugan, Phys. Rev. D 70, 124036 (2004).

[38] G. B. Cook and H. P. Pfeiffer, Phys. Rev. D 70, 104016 (2004).

[39] M. Caudill, G. B. Cook, J. D. Grigsby, and H. P. Pfeiffer, gr-qc/0605053.

[40] A. Ashtekar and G. Galloway, "Some Uniqueness Results for Dynamical Horizons" (unpublished).

[41] I. Booth and S. Fairhurst, Phys. Rev. Lett. 92, 011102 (2004).

[42] W. Kavanagh and I. Booth, gr-qc/0603074.

[43] I. Booth and S. Fairhurst, Classical Quantum Gravity 22, 4515 (2005).

[44] P. C. Vaidya, Proc. Indian Acad. Sci. A 33, 264 (1951).

[45] Y. Kuroda, Prog. Theor. Phys. 72, 63 (1984).

[46] E. Schnetter and B. Krishnan, Phys. Rev. D 73, 021502(R) (2006).

[47] I. Booth, L. Brits, J. A. Gonzalez, and C. V.D. Broeck, Classical Quantum Gravity 23, 413 (2006).

[48] J. Thornburg, Classical Quantum Gravity 21, 743 (2004).

[49] D. M. Eardley, Phys. Rev. D 57, 2299 (1998).

[50] I. Ben-Dov, Phys. Rev. D 70, 124031 (2004).

[51] J. R. Oppenheimer and H. Snyder, Phys. Rev. 56, 455 (1939).

[52] R. Schoen, in The Miami Waves Conference, 2005.

[53] A. Ashtekar, J. Engle, T. Pawlowski, and C. Van Den Broeck, Classical Quantum Gravity 21, 2549 (2004).

[54] D. S. Brill and R.W. Lindquist, Phys. Rev. 131, 471 (1963).

[55] G. B. Cook, Living Rev. Relativity 3, 5 (2000), http:// relativity.livingreviews.org/Articles/lrr-2000-5/ index.html.

[56] C. W. Misner, Ann. Phys. (N.Y.) 24, 102 (1963).

[57] B. Krishnan, Ph.D. thesis, Pennsylvania State University (2002), http://etda.libraries.psu.edu/theses/approved/ WorldWideIndex/ETD-177/index.html.

[58] M. Alcubierre and B. Brügmann, Phys. Rev. D 63, 104006
(2001).

[59] M. Alcubierre, B. Brügmann, D. Pollney, E. Seidel, and R. Takahashi, Phys. Rev. D 64, 061501(R) (2001).

[60] M. Alcubierre, B. Brügmann, P. Diener, M. Koppitz, D. Pollney, E. Seidel, and R. Takahashi, Phys. Rev. D 67, 084023 (2003).

[61] T. Goodale, G. Allen, G. Lanfermann, J. Massó, T. Radke, E. Seidel, and J. Shalf, in Vector and Parallel Processing-VECPAR'2002, 5th International Conference, Lect. Notes Comput. Sci. (Springer, Berlin, 2003); http://www.cactuscode.org/Publications/

[62] CACTUS Computational Toolkit home p., http://www. cactuscode.org/.

[63] E. Schnetter, S.H. Hawley, and I. Hawke, Classical Quantum Gravity 21, 1465 (2004).

[64] Mesh Refinement with Carpet, http://www.carpetcode.org/

[65] J. Thornburg, Phys. Rev. D 54, 4899 (1996).

[66] S. Brandt and B. Brügmann, Phys. Rev. Lett. 78, 3606 (1997).

[67] G. B. Cook, Phys. Rev. D 50, 5025 (1994).

[68] J. Baker, M. Campanelli, C. O. Lousto, and R. Takahashi, Phys. Rev. D 65, 124012 (2002).

[69] J. Baker, M. Campanelli, C. O. Lousto, and R. Takahashi, astro-ph/0305287.

[70] M. Alcubierre, B. Brügmann, P. Diener, F. S. Guzmán, I. Hawke, S. Hawley, F. Herrmann, M. Koppitz, D. Pollney, E. Seidel et al., Phys. Rev. D 72, 044004 (2005).

[71] B. Brügmann, W. Tichy, and N. Jansen, Phys. Rev. Lett. 92, 211101 (2004).

[72] M. Alcubierre, P. Diener, F. S. Guzmán, S. Hawley, M. Koppitz, D. Pollney, and E. Seidel, "Shift Conditions for Orbiting Binaries in Numerical Relativity" (unpublished).

[73] M. Ansorg, B. Brügmann, and W. Tichy, Phys. Rev. D 70, 064011 (2004).

[74] E. Schnetter, F. Herrmann, and D. Pollney, Phys. Rev. D 71, 044033 (2005).

[75] L. Baiotti, I. Hawke, P. J. Montero, F. Löffler, L. Rezzolla, N. Stergioulas, J. A. Font, and E. Seidel, Phys. Rev. D 71, 024035 (2005).

[76] L. Baiotti, I. Hawke, L. Rezzolla, and E. Schnetter, Phys. Rev. Lett. 94, 131101 (2005).

[77] C. D. Ott, H. Dimmelmeier, I. Hawke, E. Schnetter, B. Zink, E. Müller, and E. Seidel, "Fully Consistent 3D General Relativistic Rotating Stellar Core Collapse with Mesh Refinement: Comparison to 2D CFC-based Approach" (unpublished).

[78] H.-O. Kreiss and J. Oliger, Methods for the Approximate Solution of Time Dependent Problems, Global atmospheric research programme publications series No. 10 (Global atmospheric research programme publications, 1973).

[79] R. Price, Phys. Rev. D 5, 2419 (1972).

[80] M. Dafermos and I. Rodnianski, Inventiones Mathematicae 162, 381 (2005).

[81] P. Anninos, G. Daues, J. Massó, E. Seidel, and W.-M. Suen, Phys. Rev. D 51, 5562 (1995).

[82] CACTUSEINSTEIN Toolkit home p., http://www.cactuscode. org/Community/numericalRelativity/.

[83] WHISKY, EU Network GR Hydrodynamics Code, http:// www.whiskycode.org/. 
[84] E. Anderson, Z. Bai, C. Bischof, S. Blackford, J. Demmel, J. Dongarra, J. Du Croz, A. Greenbaum, S. Hammarling, A. McKenney et al., LAPACK Users' Guide (Society for Industrial and Applied Mathematics, Philadelphia, PA, 1999), 3rd ed., ISBN 0-89871-447-8 (paperback).

[85] LAPACK: Linear Algebra Package, http://www.netlib.org/ lapack/.

[86] BLAS: Basic Linear Algebra Subroutines, http://www. netlib.org/blas/.

[87] Netlib Repository, http://www.netlib.org/.

[88] W. H. Press, B.P. Flannery, S. A. Teukolsky, and W. T. Vetterling, Numerical Recipes (Cambridge University Press, Cambridge, England, 1986).

[89] UMFPACK,http://www.cise.ufl.edu/research/sparse/ umfpack/. 